\title{
Choosing Peers: Homophily and Polarization in Groups
}

\author{
Mariagiovanna BACCARA* LeEAT YARIV ${ }^{\dagger \ddagger}$
}

March 28, 2016

\begin{abstract}
This paper studies the formation of peer groups entailing the joint production of public goods. In our model agents choose their peers and have to pay a connection cost for each member added to the group. After groups are formed, each agent selects a public project to make a costly contribution to, and all members of the group experience the benefits of these contributions. Since agents differ in how much they value one project relative to the other, the group's preferences affect the generated profile of public goods. We characterize mutually optimal groups, groups that are optimal for all their members. When contribution costs are low relative to connection costs, mutually optimal groups must be sufficiently homogeneous. As contribution costs increase relative to connection costs, agents desire more connections, which in turn raises the risk of free riding. Extreme peers are then more appealing, since they are more willing to contribute, and polarization arises.
\end{abstract}

Keywords: Homophily, Peer Groups, Mutually Optimal Groups, Public Goods, Tiebout.

*Olin School of Business, Washington University in St.Louis, mbaccara@wustl.edu, (314)935-3639, http://apps.olin.wustl.edu/faculty/baccara/

${ }^{\dagger}$ Division of the Humanities and Social Sciences, Caltech, lyariv@hss.caltech.edu, (626)395-8924, http://www.hss.caltech.edu/ lyariv

${ }_{\ddagger}^{\ddagger}$ We thank Wouter Dessein, Wolfgang Pesendorfer, Alistair Wilson, Jun Zhang, the Editor, and two anonymous referees for very useful conversations and suggestions. Financial support from the National Science Foundation (SES 0963583) and the Gordon and Betty Moore Foundation (through grant 1158) is gratefully acknowledged. 


\section{INTRODUCTION}

Many social interactions entail the joint production of public goods. Clubs, associations, or online groups have members jointly initiate and organize projects and events. For instance, as of 2015, there are more than 22 million Meetup members selecting themselves into over 207, 000 social groups across 181 countries. These groups revolve around commonly planned meetings and volunteering activities. ${ }^{1}$ Student associations congregate around the organization of campus events or public service, such as women's career networking, community outreach, sexual harassment helplines, etc. Similarly, local communities in geographical neighborhoods are often the driving force behind activities such as sport tournaments or music festivals, as well as tasks such as security, gardening, cleaning, etc. and computer programming forums often entail users contributing to the development or explanation of software. ${ }^{2}$ Furthermore, most groups that are focused on a particular activity or hobby (such as biking clubs, food clubs, and so on) entail contributions to the activity itself as well as to the social interactions of the group. ${ }^{3}$

Three elements are central in these types of social interactions. First, individuals are often in the position to freely choose whom to form a community with. While this is particularly true in environments like online platforms, in which new technologies effectively eliminate geographical boundaries, it also applies to local groups - individuals can ex-ante choose which student association to join within their campus, which neighborhood to live in, etc. Second, contribution to the production of public goods is costly. Therefore, strategic considerations, namely the possibility of free riding, come into play. Third, connecting to other individuals often entails a cost. The marginal cost of an additional connection can display different dependencies on the group's size. It could be increasing in group size if groups face congestion

\footnotetext{
${ }^{1}$ There are approximately 550,000 monthly Meetup events. Over 3,000 of the groups focus explicitly on public goods in the form of volunteering, see http://www.meetup.com/about/ and http://volunteering.meetup.com/

${ }^{2}$ Indeed, many open-source programming resources have substantial voluntary contributions from individuals. A prime example is the development of Linux. For instance, when looking at contributors to the Linux Kernel since version 2.6.36, approximately $25 \%$ of contributions were done by developers who were not paid for their work, see the "Linux Kernel Development Report," published in 2012 by the Linux Foundation.

${ }^{3}$ In fact, such activities and hobbies often have several aspects individuals can contribute to. For instance, in a biking club, contributions can take the form of carrying first-aid kits, participating in a pace line, mapping new routes, and so on. In a food club, members can contribute to different types of cooking, e.g. baking and grilling.
} 
externalities: for example, if a student association grows in size, holding meetings may become more difficult. Other times, adding one connection involves a constant cost, possibly no cost, up to a fixed capacity: for example, building cooperatives involve fixed membership fees and are bound by real-estate constraints.

These three elements jointly determine the size and composition of social communities, as well as their performance in terms of the public goods they ultimately yield. This paper's goal is to illustrate how the interplay between public-good production and connection technologies affects the patterns exhibited by social groups.

We study a model in which agents make costly contributions to two different public projects or tasks. Each agent's preferences are characterized by a parameter in $[0,1]$, measuring how much she cares about one project relative to the other. For simplicity, we assume that each individual can make a contribution to at most one project. For example, a university student joining an association may have limited time and therefore face a choice of which of the association's missions to actively pursue. Before making contributions, agents have the possibility of forming groups. What defines a peer group is that all its members benefit from the contributions made within the group towards the public projects. In addition, each individual in a group has to pay a cost to connect with each other member. We assume that overall connection costs are increasing and convex in the size of the group. We allow agents to select the number as well as the types of their peers. We consider a group "mutually optimal" if it is optimal for all of its members. ${ }^{4}$

In this general setting, when contribution costs are large compared to connection costs or, equivalently, connection costs are persistently low, agents desire more connections. However, such expansion introduces the risk of free-riding. Indeed, there is a risk of individuals joining a group in order to enjoy the contributions of others, without contributing themselves. More extreme individuals, ones who care about one of the projects far more than the other, have greater incentives to contribute to that project. Therefore, introducing group polarization (i.e., including extremists on both tasks) has the benefit of mitigating the free-rider problem

\footnotetext{
${ }^{4}$ This notion is in the spirit of the appoach introduced in Baccara and Yariv (2013), with one important distinction: in Baccara and Yariv (2013) we fix group size, and we allow agents to choose their group's composition only. Here the group size is endogenous and agents are allowed to select both the group size and their peers' types.
} 
by weakening the incentive constraints pertaining to project contributions. Mutual optimality requires both types of extremists to desire connections with one another. Polarized groups are then mutually optimal only if connection costs are low enough. Whenever connection costs are positive, no free riding can occur in a mutually optimal group - free riders come at a cost but provide no benefit. It follows that for small but positive connection costs, mutually optimal groups are formed only by extremists on both dimensions. Each sub-group of extremists contributes to the dimension it cares most about. Take the example of student associations and suppose contribution costs are substantial (due to the time commitment they require), while connection costs are significant but small (especially relevant after the introduction of electronic messaging platforms). We then expect all members to actively contribute to one of the missions of the association. Our results also suggest extremist members, ones very dedicated to the mission they contribute to, though not necessarily to other missions pursued by the association.

Since the driving force behind the emergence of mutually optimal polarization is the freerider problem, reducing contribution costs plays a similar role to increasing connection costs: they both alleviate the potential for free riding. Indeed, when contribution costs are small compared to connection costs, the number of individuals that agents desire to connect to is relatively small. The resulting groups are then not at risk of free riding. In this case, mutual optimality entails agents agreeing on how large the group should be, and on how to distribute optimally contributions across the two tasks. Therefore, mutually optimal groups are fairly homogenous. They can, however, entail both diversification of projects or specialization: if members are moderate, the group generates contributions to both projects; if members are extremists who all care far more about one of the projects, the group specializes and contributions are all made toward that project.

When contribution costs are relatively low, mutually optimal groups may also entail a mild degree of polarization. Such groups include two subsets of agents. Each subgroup cares more about a different task and contributes to that task. Nonetheless, agents in both subsets are similar enough so that they agree on the group's size.

In the student association example, suppose contribution costs are not too high (since the activities the association pursues are not very time demanding to organize), but connection 
costs are substantial (say, when considering associations formed prior to the introduction of the Internet). In this case, when we see non-trivial contributions to several different missions, our results indicate that the group is fairly homogeneous and contains moderate members, individuals who care about all of the missions supported under the umbrella of the association.

Our results yield a set of predictions on the types of agents that cannot be part of any non-trivial mutually optimal group, one with at least two members. If contribution costs are large compared to connection costs, our analysis implies that non-extreme individuals cannot be part of a mutually optimal group when connection costs are positive. Indeed, in this case moderate individuals would free ride on others were they to join a group. Consequently, no agent is willing to pay a positive connection cost to associate with them. On the other hand, when contribution costs are small compared to connection costs, the incentives of moderate individuals to contribute in mutually optimal groups can be substantial. In this case, the participation in mutually optimal groups is determined by the magnitude of connection costs. We show that for sufficiently small connection costs, individuals of all types are part of nontrivial mutually optimal groups. For high connection costs, some individuals may prefer to avoid any prohibitively costly association with others. Therefore, the only mutually optimal groups such individuals can be part of are singletons.

We demonstrate the implications of two special cases of connection costs. First, we consider step-function connection costs, which are zero up to a fixed group size, and very high for larger groups. For such costs, there is a set of moderate taste parameters such that individuals with those tastes can only be part of mutually optimal groups that are either sufficiently small (in which all members have incentives to contribute) or very large (in which the moderate agents free ride on the extremists in the group, who make all contributions). Second, we consider linear connection costs. For these costs, we show that for a fixed level of contribution costs, the homogeneous groups arising for high connection costs are smaller than the polarized groups arising for low connection costs. That is, there is a positive correlation between group size and heterogeneity.

Our results provide a taxonomy for mapping different varieties of technological improvements into changes in socialization patterns. A decrease in the contribution cost may occur with the development of a new technology that makes public good production more efficient. 
Thus, our results suggest that as public good technologies improve (while connection costs stay constant), mutually optimal groups exhibit more similarity in tastes. For example, in residential neighborhoods, which are characterized by fixed capacity constraints, as public project contributions become cheaper (e.g., communication technologies make event organization more efficient), residents would be expected to display increasingly similar preferences. On the other hand, as connection technologies improve and congestion externalities decrease (while contribution costs stay constant) the free-rider problem becomes more severe and more polarization tends to emerge in mutually optimal groups. Naturally, some new technologies tend to lower both connection and contribution costs. In the academic context, while E-mail or Skype have significantly reduced connection costs, the introduction of search engines has cut back on contribution costs. Our analysis provides a framework to assess which force is the dominant one in affecting the structure of mutually optimal groups. ${ }^{5}$

Related Literature. This paper has some elements in common with several literature strands. First, there is a vast literature in economics studying different aspects of public goods interactions (see, e.g., Ledyard (1995) for a review). This literature has not looked at how the link between the ex-ante choice of peers and the ex-post public goods production influences socialization patterns.

A rich literature in urban economics, descending from Tiebout (1956), addresses the link between neighborhood sorting and public goods. This line of work has addressed, for example, the effect of improvements in public goods' provision on communities' density and diversity, the link between the types of public goods chosen to be offered and community sorting, and so on. ${ }^{6}$ In this literature, however, the provision of public goods is either exogenous, or set at the efficient level given the preferences represented in the group. In addition, the concept of mutual optimality used is typically a myopic one, in which each individual does not anticipate

\footnotetext{
${ }^{5}$ Rosenblat and Mobius (2004) studied coauthored papers in top economics journals between the years 19691999. They showed how the introduction of the Internet in the early 1990's is linked with a $20 \%$ decrease in the realization of projects with a dissimilar coauthor. These would suggest that the reduction of contribution costs was dominant with respect to that on connection costs. See also Sproull and Kiesler (1991) for the impacts of new technologies on social connections.

${ }^{6}$ For example, it has been empirically documented how improvements in public goods tend to increase the size of a community (Banzhaf and Walsh, 2008). See also Rhode and Strumpf (2003) and Sethi and Somanathan (2004).
} 
changes in the public good offerings when contemplating a deviation to a different community. As we consider public goods directly contributed by the members of the community, we take a non-cooperative approach to public goods' provision. Also, we assume that individuals anticipate the equilibrium public good provision that will arise upon their deviation to a different group. ${ }^{7}$

Several recent papers address preferences for similarity. Currarini, Jackson, and Pin (2009) assume homophilous behavior and study its consequences in a friendship formation model. Peski (2008) derives an endogenous preference for similarity by assuming some properties of preferences on friendship and the possibility of confusing people who are similar to each other. Kets and Sandroni (2015a,b) suggest theory of mind as a mechanism driving similar individuals to predict better each other's actions. While similarity within groups may therefore be useful for achieving coordination at high rates, it does not always yield the most efficient coordination. In particular, both diverse and homogenous groups can be optimal, depending on the payoff environment. Finally, in Baccara and Yariv (2013), we consider a model in which agents, who differ in how much they value one task relative to another, choose their peers before making free contributions to public tasks. We show that when contributions are free, mutually optimal groups must be sufficiently homogeneous. The current paper differs from Baccara and Yariv (2013) in its main focus: it aims to capture applications such as communities and neighborhoods in which individuals volunteer costly contributions to public projects. The difference in focus translates into two important differences between the underlying models of the two papers. First, in the current paper contributions to public projects are costly rather than free, thereby generating the potential for free riding. Second, whereas in Baccara and Yariv (2013) group size is always exogenously given, here we allow agents to select any number of peers they desire. This allows us to derive predictions on the size of mutually optimal communities, and on the correlation between size and other group features such as taste heterogeneity.

\footnotetext{
${ }^{7}$ Robbett $(2013,2014)$ provides observations from an array of experiments that allow individuals with heterogenous preferences to dynamically move between communities in which a public goods game is later played, considering different institutions that determine the level and enforcement of individual contributions. She illustrates how chronic movements between communities may persist, that high connection fees in a location attract individuals of a particular type, and that voting on public goods provision helps in terms of utilitarian efficiency.
} 
The underlying idea that the group of players in a strategic situation is, in itself, endogenous motivates some of the work on club formation (see, e.g., Ellickson, Grodal, Scotchmer, and Zame (1999)). The basic model of that literature assumes some form of externality across individuals and studies endogenous group formation (often in a general equilibrium setup) in the presence of these externalities. Our paper, by addressing group formation and strategic interaction at the same time, provides a foundation for a class of externalities of this sort. Furthermore, our analysis allows us to characterize the properties (such as homogeneity, polarization, etc.) of the endogenous groups.

Finally, the idea that agents' preferences may alleviate incentive constraints in collective settings with costly contributions appears in some recent mechanism design literature. Indeed, Che and Kartik (2008) and Gerardi and Yariv (2008) illustrate how a designer may optimally choose a committee of experts that are more extreme than she in order to achieve more information revelation and higher quality decisions.

\section{The Model}

Consider two tasks, $A$ and $B$. A group consists of multiple agents, and contributions to tasks are public within each group. For instance, groups can stand for student associations, neighborhood committees, a collection of friends, etc. In these sorts of groups, members can contribute in different ways. In student associations, members can contribute to university social events or community outreach; in neighborhood committees, members can organize church activities, social events, etc.; in interactions with friends, individuals can get informed on different joint interests such as new movies or new restaurants.

Specifically, each agent in a group needs to decide which task to contribute to or whether to forego any contribution. That is, each agent chooses an action $v \in\{A, B, \varnothing\}$. There are two production functions, $f_{A}$ and $f_{B}$, that map contributions into utilities derived from either task. Suppose that $f_{A}$ and $f_{B}$ are defined over $\mathbb{R}$ and are strictly increasing and concave. Agents differ in how much they care about either task. Thus, the general utility for an agent

of taste $t \in[0,1]$ who is in a group of $n$ agents, $k^{A} \leq n$ of whom contribute to task $A$ and $k^{B} \leq n-k^{A}$ of whom contribute to task $B$, is given by:

$$
U\left(t, k^{A}, k^{B}\right) \equiv t f_{A}\left(k^{A}\right)+(1-t) f_{B}\left(k^{B}\right) .
$$


The idea behind the assumption that each agent can make at most one contribution is that each agent has a limited budget of resources to allocate to public projects. ${ }^{8}$ We assume that contributing to tasks is costly: making a contribution to either task comes at a cost of $c \geq 0$. The cost $c$ is strictly positive in any context in which contributions require effort. An agent who decides to forego contributions does not incur any cost.

We assume that in any group of size $n \geq 1$ each agent pays a connection cost $D(n)$. We assume that $D(1)=0$, so that an agent incurs no connection costs when remaining alone. The marginal cost of connecting to an additional individual will play an important role in our analysis. We denote this marginal cost by $d(1) \equiv 0, d(n) \equiv D(n)-D(n-1) \geq 0$ for all $n \geq 2$ and assume $d(n)$ is (weakly) increasing for all $n$. That is, connection costs are increasing and convex in the size of the group one is a member of. For presentation simplicity, we will assume that $d(n) \neq c$ for all $n .^{9,10}$

For any group of size $n$ composed of agents with tastes $\left(t_{1}, \ldots, t_{n}\right)$, expected payoffs are ultimately identified by the profile of chosen tasks $\left(x_{1}, \ldots, x_{n}\right)$, where $x_{i} \in\{A, B, \varnothing\}$ is the action chosen by agent $i$. We call the induced game the task-selection game. As a tiebreaking rule, we assume that an agent who is indifferent between tasks $A$ and $B$ makes an $A$ contribution, and an agent who is indifferent between contributing and not contributing makes a contribution (this simplifies the exposition, but is not crucial for our analysis). We focus on equilibria in pure strategies. As it turns out, given our tie-breaking rule, pure equilibria exist. Throughout the paper, we assume that the most (utilitarian) efficient such equilibrium is selected. As it turns out, there is a simple characterization of an efficient equilibrium.

Lemma 1 (Existence) For any group of $n$ agents with tastes $t_{1} \geq t_{2} \geq \ldots \geq t_{n}$, there exists $\tau^{A} \in\{0, \ldots, n\}$ and $\tau^{B} \in\{1, \ldots, n+1\}, \tau^{B}>\tau^{A}$, such that all agents $i \leq \tau^{A}$ making an A-contribution, all agents $i \geq \tau^{B}$ making a B-contribution, and all other agents not making any contribution, constitutes an efficient Nash equilibrium of the task-selection

\footnotetext{
${ }^{8}$ The analysis of the paper does not change qualitatively if we assume that each agent can make any fixed number of contributions $h \geq 1$.

${ }^{9}$ This assumption is not crucial for the analysis, but does rule out knife-edge cases in which an agent is indifferent between adding an additional member to her group and not doing so when that additional member would make a contribution instead of the agent herself.

${ }^{10}$ Several important elements distinguish this model from the one presented in Baccara and Yariv (2013). Most notably, in Baccara and Yariv (2013) contributions are free $(c=0)$ and, for the most part, group size is fixed, corresponding to the step-function connection costs case addressed later in Sections 4 and 5 .
} 
game. Furthermore, the volume of $A$ - and B-contributions corresponding to an efficient Nash equilibrium outcome is uniquely determined.

Given a group of $n$ agents with tastes $t_{1} \geq t_{2} \geq \ldots \geq t_{n}$, Lemma 1 allows us to concentrate on equilibria $\left(x_{1}, \ldots, x_{n}\right)$ identified by two thresholds $\tau^{A} \in\{0, \ldots, n\}$ and $\tau^{B} \in\{1, \ldots, n+1\}$ such that $x_{1}=\ldots=x_{\tau^{A}}=A$ and $x_{\tau^{B}}=\ldots=x_{n}=B$ (in particular, if $\tau^{A}=n$, all agents choose an $A$-contribution, and if $\tau^{B}=1$, all agents choose a $B$-contribution). In words, we focus on equilibria in which any agent choosing task $A$ cares more about that task than does any agent not choosing any contribution or choosing task $B$. We call such equilibria ordered. With our tie-breaking rule, Lemma 1 guarantees that ordered equilibria are the most utilitarian efficient among Nash equilibria of the task-selection game (though there may be other equally efficient equilibria).

In the proof of Lemma 1 we show that equilibrium outcomes in a group of size $n$ are ranked according to the volume of contributions made on each task. That is, whenever more $A$-contributions are made in one equilibrium relative to another, more $B$-contributions will be made as well in that former equilibrium. As a consequence, all agents agree that the efficient equilibrium is the most preferred.

It is important to note that task-selection equilibria may involve agents not making contributions. The fact that contributions are costly introduces a free-rider problem. Indeed, in any equilibrium in which $\tau^{B}>\tau^{A}+1$, the agents $i, \tau^{A}<i<\tau^{B}$, do not have enough incentives to make contributions to either task. Of course, if $c=0$, the free-rider problem disappears and all agents contribute in equilibrium (i.e., $\tau^{B}=\tau^{A}+1$ ).

We also note that as long as any group of $n$ agents has distinct tastes $t_{1}>t_{2}>\ldots>t_{n}$, there is a unique ordered equilibrium. However, if some participants' tastes coincide, there may be multiple equilibria. This multiplicity takes a simple form - suppose that $t_{j}=t_{j+1}=$ $\ldots=t_{j+k}$ for some $j, k$. If only $k^{\prime}<k$ agents with taste $t_{j}$ are to contribute in an ordered equilibrium, their identity is not pinned down. In this case, any selection of $k^{\prime}$ of the $k$ agents would correspond to an ordered equilibrium. In particular, while the distribution of ordered equilibrium payoffs is determined uniquely, individual payoffs could vary across equilibria. 


\section{Mutually Optimal Groups}

Let us consider an extended game composed of two stages. First, each agent of taste $t \in[0,1]$ can select the size as well as the composition of her peer group. ${ }^{11}$ Second, the task-selection game described above is played within the chosen group. As mentioned, we assume that an ordered equilibrium arises in this stage.

Lemma 1 guarantees that the number of $A$ - and $B$-contributions are determined uniquely in any ordered equilibrium of the task-selection game, but there might still be multiple such ordered equilibria. In principle, when contribution costs are high relative to connection costs, an extremist agent who contributes in one group might prefer a larger group of identical extremists, one of size exceeding the maximal possible number of contributions, in the hopes of having extremists other than herself contribute. In order to circumvent such behavior that is due to a perceived positive resolution of coordinated contributions, we assume that agents evaluate groups they do not belong to based on the worst possible payoff they can obtain in any equilibrium of the task-selection stage played by the group. This is tantamount to individuals being pessimistic about the solution to any coordination problem involving a subset of agents contributing.

Formally, for any group with taste profile $\mathbf{t}=\left(t_{1}, \ldots, t_{n}\right)$, let $\underline{u}_{i}(\mathbf{t})$ denote the minimal possible payoff agent $i$ receives in any equilibrium, not necessarily ordered, of the task-selection game. We define mutual optimality in the first stage of the extended game as follows.

Definition (Mutually Optimal Group) A group of $n$ agents is mutually optimal if there exists an ordered equilibrium in the task-selection game with utility profile $\left(u_{1}, \ldots, u_{n}\right)$ such that for any agent $i$, for any other group with taste profile $\mathbf{t}^{\prime}=\left(t_{1}^{\prime}, \ldots, t_{n^{\prime}}^{\prime}\right)$ that includes $i, u_{i} \geq \underline{u}_{i}\left(\mathbf{t}^{\prime}\right)$.

In a mutually optimal group, each agent maximizes her expected utility, foreseeing the equilibrium played in the task-selection game that ensues. ${ }^{12}$ In relation to agents' pessimistic

\footnotetext{
${ }^{11}$ This amounts to assuming that the pool of potential agents to choose from is infinite and that any combination of tastes is feasible.

${ }^{12}$ Our notion of mutual optimality here resembles that of stability in Baccara and Yariv (2013). We thank an anonymous referee for inspiring this label in order to distinguish our solution concept from that used by the cooperative game theory literature. Indeed, notice that cooperative solutions usually restrict agents' options by considering only feasible deviations. In our case, this would translate to agents considering the menu of
} 
beliefs in groups other than their own, two remarks are in order. First, this restriction on beliefs assures potential deviations are as unappealing as reasonably possible and therefore makes mutual optimality easier to achieve. Even with this seemingly weak restriction, we will show that mutually optimal groups exhibit very particular features. Second, our analysis will show that requiring only extreme agents, those who care sufficiently much about one of the two tasks, rather than all agents in a group, to hold such pessimistic beliefs would generate an identical characterization to the one presented here. ${ }^{13}$

This notion of mutual optimality is a natural equilibrium condition for the group-selection stage in an environment that allows individuals in large populations to connect in an unconstrained way. ${ }^{14}$ For example, university students have a wide choice of which association to join, or to form together with their peers. A student association is then mutually optimal if all of its active members are satisfied with the set of other participants.

The goal of the rest of this section is to analyze some properties of group composition entailed by the mutual optimality notion introduced above. The following definitions are useful for our analysis.

First, denote by $m^{A}(n ; t)$ the optimal number of $A$-contributions an agent with taste groups that would admit them as members. The notion of mutual optimality does not require us to take a stand on how groups determine whether to admit a new member. In particular, our mutually optimal groups would be "stable" under any protocol specifying which groups would be willing to add which types of agents.

${ }^{13}$ In fact, all our results would remain qualitatively similar were we to assume that agents have more optimistic beliefs, predicting an equilibrium will be selected uniformly at random from the set of all equilibria in the task-selection game. That way, coordination on whom should contribute is resolved by randomization. That is, after committing to their group, if there are $m$ individuals in a group with tastes in some interval such that each would be willing to contribute up to the $k$-th unit corresponding to one task, where $k \leq m$, then ultimately $k$ out of the $m$ are chosen randomly to contribute. We maintain our assumption regarding the agents' pessimism since it simplifies the description of our results (and return to the one modification the above alternative assumption would imply in Footnote 17).

${ }^{14}$ In the current paper we do not consider population-wide equilibria. However, one could certainly contemplate such a notion. Suppose a population is characterized by a distribution of tastes. One way to think of a population-equilibrium is to consider partitions of the population into groups such that each individual prefers her own group to joining any other element of the partition. Whenever the population can be partitioned into mutually optimal groups, an equilibrium exists. In that respect, our results suggest for which population distributions the existence of an equilibrium as such is guaranteed. Another way to think of a populationequilibrium that circumvents any existence issues is to consider partitions of the population into groups such that each group is either mutually optimal or a singleton. In this case, a population-equilibrium in which all individuals remain as singletons always exists. Our analysis suggests when other equilibria might arise, ones with non-trivial mutually optimal groups. We also describe the features of such groups as a function of the underlying characteristics of the environment. 
parameter $t$ would choose out of a total of $n$ available contributions. That is, given $t \in[0,1]$, $m^{A}(n ; t)$ is the maximal integer $h \leq n$ such that

$$
U(t, h, n-h) \geq U(t, h-1, n-h+1)
$$

is satisfied. ${ }^{15}$ If $(1)$ is not satisfied for any $h$, we define $m^{A}(n ; t)=0$. Let $m^{B}(n ; t) \equiv n-$ $m^{A}(n ; t)$.

Absent contribution costs, were groups fixed at a size of $n$, mutually optimal groups would be comprised of $n$ individuals who all agree on the allocation of contributions to tasks. That is, mutually optimal groups would take the form of $\left\{i_{1}, \ldots, i_{n}\right\}$, where $i_{j}$ is of type $t_{j}$ such that $m^{A}\left(n ; t_{l}\right)=m^{A}\left(n ; t_{k}\right)$ for all $l, k=1, . ., n$. In fact, Baccara and Yariv (2013) focuses precisely on this case and illustrates that a group is mutually optimal if and only if all taste parameters in the group belong to the same element of a partition $\left\{T_{k}^{n}\right\}_{k=0}^{n}$ of the interval $[0,1]$. Indeed, any element $T_{k}^{n}$ in the partition includes all taste parameters of agents who agree on the optimal allocation of $n$ contributions across the two dimensions. This benchmark case ends up playing a role in the general characterization of mutually optimal groups we provide below.

Next, for $x=A, B$, and for any $s>0$, let $k^{x}(s ; t)$ denote the maximal number of $x$ contributions made in a group for which an agent of taste $t$ is willing to acquire an $x$ contribution at a cost $s$. In particular, $k^{x}(s ; t)$ is the maximal number of $x$-contributions, each costing $s$, an agent of taste $t$ would be willing to make were she acting on her own and able to make more than one contribution. Alternatively, $k^{x}(s ; t)-1$ is the maximal number of individuals the agent would be willing to connect with at a cost $s$ when she, together with these individuals, make an $x$-contribution. Formally, for any $s>0, k^{A}(s ; t)$ is the maximal integer $h$ such that, no matter how many $w B$-contributions are acquired,

$$
U(t, h, w)-U(t, h-1, w)=t\left[f_{A}(h)-f_{A}(h-1)\right] \geq s .
$$

Similarly, for any $s>0, k^{B}(s ; t)$ is defined as the maximal integer $h$ such that, no matter how

\footnotetext{
${ }^{15}$ Under our assumptions on $f_{A}$ and $f_{B}$, if inequality (1) is satisfied for $h$, it is satisfied for any $h^{\prime}<h$.
} 
many $w$ A-contributions are made,

$$
U(t, w, h)-U(t, w, h-1)=(1-t)\left[f_{B}(h)-f_{B}(h-1)\right] \geq s .
$$

When $s=0$, we denote $k^{A}(0 ; t)=k^{B}(0 ; t)=\infty$, corresponding to each agent's willingness to acquire a free contribution regardless of the number of contributions already available. For any $t \in[0,1]$, we define $k(s ; t) \equiv k^{A}(s ; t)+k^{B}(s ; t)$ as the total attainable number of contributions at a cost $s$ for an agent of taste $t$. At different points of the analysis, depending on whether agents consider contributions made by themselves or by other group members, $s$ will stand for either the contribution $\operatorname{cost} c$, or a connection $\operatorname{cost} d(l)$ for some $l \geq 1$.

Next, let $k_{\max }^{A}(s)$ be the maximal attainable number of $A$-contributions at a cost $s$ that corresponds to agents with the most extreme taste parameter $t=1$. That is, $k_{\max }^{A}(s) \equiv$ $k^{A}(s ; 1)$. Analogously, $k_{\max }^{B}(s) \equiv k^{B}(s ; 0)$. Note that for any $s \geq 0, k^{A}(s ; t)$ is increasing in $t$ and $k^{B}(s ; t)$ is decreasing in $t$. Taking $s=c, k_{\max }^{A}(c)$ and $k_{\max }^{B}(c)$ are the maximal number of $A$ - and $B$-contributions that can be expected to be made, respectively, in any equilibrium of the task-selection game.

Finally, let an $A$-extremist be an agent of taste $t$ such that $k^{A}(c ; t)=k_{\max }^{A}(c)$. In words, $A$-extremists are agents who are willing to make the maximal number of $A$-contributions. Likewise, $B$-extremists are agents of taste $t$ such that $k^{B}(c ; t)=k_{\max }^{B}(c)$. It is easy to see that $A$ - and $B$-extremists are agents with "sufficiently extreme" tastes to be willing to make the maximal possible number of contributions on the task they care about, and their tastes lie in two intervals of the form $[\underline{t}, 1]$ and $[0, \bar{t}]$, respectively.

Our goal is to analyze the properties of mutually optimal groups for general connection costs $D(\cdot)$. First of all, we note the existence of mutually optimal groups:

Lemma 2 (Existence of Mutually Optimal Groups) For any production functions, contribution cost $c$, and connection costs $D(\cdot)$, mutually optimal groups exist. That is, there exist $t_{1}, \ldots, t_{\tilde{n}} \in[0,1]$ such that the group composed of $\tilde{n}$ individuals with taste profile $\left(t_{1}, \ldots, t_{\tilde{n}}\right)$ is mutually optimal.

To see that mutually optimal groups always exist, consider for example a group of identical individuals of taste $t=1$, where members are added up to the point in which, by 
introducing one additional member, either that member does not have incentives to make an $A$-contribution any longer, or does have incentives to contribute, but the marginal utility from her contribution is exceeded by the connection cost required to add her to the group. This group would be finite as long as $c>0$ or $d(n)>0$ for some $n$ and infinite otherwise. It is easy to see that it is mutually optimal. A similar mutually optimal group can be constructed by considering identical individuals of taste $t=0$. When contribution costs and connection costs are low, non-trivial mutually optimal groups exist. When contribution costs or connection costs become prohibitively high, the only mutually optimal groups are singletons.

Our goal is to characterize all non-trivial (i.e., containing at least two people) mutually optimal groups. We show that the structure of mutually optimal groups depends crucially on the relative size of contribution and connection costs with respect to one another. Denote by $\widetilde{n}(c)$ the maximal integer such that $d(n)<c .{ }^{16}$ If $d(n)<c$ for all $n$, we denote $\widetilde{n}(c)=\infty$. Therefore, $\widetilde{n}(c)$ represents a critical size of a group above which connecting to additional members becomes more costly than contributing.

As $c$ increases, the maximal number of attainable contributions that can conceivably be made in equilibrium, $k_{\max }^{A}(c)+k_{\max }^{B}(c)$, decreases. Moreover, as $c$ increases, $\widetilde{n}(c)$ increases as well. Let $c^{*} \equiv \sup \left\{c \mid k_{\max }^{A}(c)+k_{\max }^{B}(c)>\widetilde{n}(c)\right\}$ and $c^{* *} \equiv \inf \left\{c \mid k_{\max }^{A}(c)+k_{\max }^{B}(c)<\widetilde{n}(c)\right\}$. Therefore, we have:

Lemma 3 (Contribution Costs Thresholds) $c^{*}$ and $c^{* *}$ are such that $k_{\max }^{A}(c)+k_{\max }^{B}(c)>$ $\widetilde{n}(c)$ for all $c \leq c^{*}$ and $k_{\max }^{A}(c)+k_{\max }^{B}(c)<\tilde{n}(c)$ for any $c>c^{* *}$. Moreover, if $c^{*} \neq c^{* *}$, $k_{\max }^{A}(c)+k_{\max }^{B}(c)=\widetilde{n}(c)$ for any $c \in\left(c^{*}, c^{* *}\right]$.

The thresholds $c^{*}$ and $c^{* *}$ determine the interplay between connection and contribution costs. If the contribution cost $c$ is above $c^{* *}$, then $k_{\max }^{A}(c)+k_{\max }^{B}(c)$, the maximal volume of contributions that can be achieved in any group, is strictly lower than $\widetilde{n}(c)$. Therefore, the maximal attainable volume of contributions can be achieved before the constraints imposed by connection costs become binding. The size of mutually optimal groups in this case has to be smaller than $\widetilde{n}(c)$. If $c$ is (weakly) below $c^{*}, k_{\max }^{A}(c)+k_{\max }^{B}(c)$ is above $\widetilde{n}(c)$. This implies that when agents consider adding agents to their group to gain more contributions, the constraints

\footnotetext{
${ }^{16}$ Recall our assumption that $d(n) \neq c$ for all $n$. It then follows that $d(\tilde{n}+1)>c$.
} 
imposed by the connection costs will bind before the maximal volume of contributions is achieved. In this case, the size of mutually optimal groups will be at least $\widetilde{n}(c)$. Finally, there could be a region between $c^{*}$ and $c^{* *}$, where $k_{\max }^{A}(c)+k_{\max }^{B}(c)$ coincides with $\tilde{n}(c)$. In this region, the constraints associated to connection costs and contribution costs bind for the same group size.

In what follows, we divide our analysis into the case of high contribution costs $\left(c>c^{* *}\right)$ and low contribution costs $\left(c \leq c^{*}\right)$.

\section{High Contribution Costs}

In this section we consider high contribution $\operatorname{costs} c$ satisfying $c \geq c^{*}$. The main message of this section is that when contribution costs are high, mutually optimal groups always contain extremist agents who achieve the maximal attainable contributions on the task they care most about.

We start by focusing on $c>c^{* *}$, which implies through Lemma 3 that $k_{\max }^{A}(c)+k_{\max }^{B}(c)<$ $\widetilde{n}(c)$. The corresponding non-trivial mutually optimal groups are characterized in the following proposition:

Proposition 1 ( High $c$ - Mutually Optimal Groups) If $c>c^{* *}$, non-trivial mutually optimal groups take one of the following forms:

1. A polarized group with $k_{\max }^{A}(c)$ A-extremists, each with a taste $t$ satisfying

$$
k^{B}\left(d\left(k_{\max }^{A}(c)+k_{\max }^{B}(c)\right) ; t\right) \geq k_{\max }^{B}(c)
$$

and $k_{\max }^{B}(c) B$-extremists, each with a taste $t$ satisfying

$$
k^{A}\left(d\left(k_{\max }^{A}(c)+k_{\max }^{B}(c)\right) ; t\right) \geq k_{\max }^{A}(c)
$$

2. A group with $k_{\max }^{x}(c) x$-extremists, each with a taste of $t$ satisfying

$$
k^{y}\left(d\left(k_{\max }^{x}(c)+1\right) ; t\right)=0, y \neq x
$$


The first part of Proposition 1 describes mutually optimal groups in which the number of contributions gathered in the task-selection phase is maximized. When $c$ is large, the maximal number of contributions in the task-selection phase is small. Therefore, forming a group that would allow for the maximal number of contributions entails connecting to a fairly small number of individuals, corresponding to low connection costs. The only way to achieve this volume of contributions is to have a group in which at least $k_{\max }^{A}(c)$ agents are $A$-extremists and at least $k_{\max }^{B}(c)$ agents are $B$-extremists. To make this group mutually optimal, it is necessary for the extremists on both sides to be willing to pay the connection costs required to obtain contributions on both dimensions. This is possible only if participating in a group with $k_{\max }^{A}(c)+k_{\max }^{B}(c)$ members is sufficiently cheap (i.e., $d\left(k_{\max }^{A}(c)+k_{\max }^{B}(c)\right)$ is lower than the marginal benefit an extremist on one dimension receives from the last contribution made toward each task). By the definition of $\widetilde{n}(c)$ and since $k_{\max }^{A}(c)+k_{\max }^{B}(c) \leq \widetilde{n}(c)$, we have $d\left(k_{\max }^{A}(c)+k_{\max }^{B}(c)\right) \leq d(\widetilde{n}(c))<c$, which guarantees that an extremist would not want to reduce the number of members contributing to the task she contributes to. We also need to assure that an extremist, say an $A$-extremist of type $t$, does not want to reduce the number of $B$-extremists in the group. The connection costs saved by reducing one $B$-extremist are given by $d\left(k_{\max }^{A}(c)+k_{\max }^{B}(c)\right)$. Therefore, we need to assure that the $A$-extremist of type $t$ is willing to invest in at least $k_{\max }^{B}(c) B$-contributions when the cost of each such contribution is effectively $d\left(k_{\max }^{A}(c)+k_{\max }^{B}(c)\right)$. This is tantamount to the restriction $k^{B}\left(d\left(k_{\max }^{A}(c)+k_{\max }^{B}(c)\right) ; t\right) \geq k_{\max }^{B}(c)$. Similar arguments generate the analogous restriction on the tastes of $B$-extremists in such polarized groups. Notice that this restriction is easier to satisfy when connection costs are low relative to contribution costs.

In the second part of Proposition 1 we describe groups in which all agents have the same, extreme, taste parameter. This is a knife-edge case in which the maximal attainable number of contributions is collected toward the task all agents care about and agents' preferences are so extreme that no one desires contributions toward the other task. ${ }^{17}$

\footnotetext{
${ }^{17}$ Some aspects of Proposition 1 depend on the assumption that agents hold pessimistic beliefs regarding equilibrium outcomes in other groups. Under the alternative assumption that any coordination problem is resolved randomly, for part (1) of Proposition 1 to hold we would need an additional condition guaranteeing that the benefit of adding members in excess of $k_{\max }^{A}(c)+k_{\max }^{B}(c)$ to a polarized group (to decrease the probability of contributing) is overwhelmed by the cost of connecting with them. The groups described in part (2) of Proposition 1 would still be mutually optimal, and possibly contain more than $k_{\max }^{x}(c)$ individuals.
} 
In the Appendix, we also show that, in fact, the groups identified by the proposition are the only possible non-trivial mutually optimal groups for $c>c^{* *}$. Nonetheless, when contribution and connection costs are sufficiently high, some moderate agents may prefer to remain singletons and not contribute to either task. In other words, trivial groups composed of one moderate individual may constitute mutually optimal groups.

If $c^{*} \neq c^{* *}$ and $c \in\left(c^{*}, c^{* *}\right]$, whenever either $k_{\max }^{x}(c)>1$ for $x=A, B$, or the connection cost function $d(n)$ is strictly positive for any $n \geq 2$, the groups described in Proposition 1 are still the only mutually optimal ones. However, if $d(n)$ is a step-function (as discussed later in this section), and $k_{\max }^{x}(c)=1$ for some $x$, say $A$, the full characterization of the mutually optimal groups includes additional types of groups consisting of $k_{\max }^{B}(c)$ agents on the $t=0$ extreme and one moderate agent. Since the full characterization of mutually optimal groups for this case involves some minor technical subtleties without adding qualitative novelties, we refer the interested reader to Proposition 1A presented in the Appendix.

Notice that absent connection costs, a positive contribution cost would imply that for sufficiently large group sizes, some individuals will ultimately not contribute. Such groups can be mutually optimal as long as the free-riding individuals come at no cost. Free riding cannot occur in mutually optimal groups whenever connection costs are strictly positive. ${ }^{18}$ In fact, as long as any member in addition to those who necessarily contribute comes at a cost, there will be no free riding in mutually optimal groups. We therefore have the following:

Corollary (High $c-$ No Free Riding) Whenever $c>c^{*}$ and $d\left(k_{\max }^{x}(c)+1\right)>0$ for $x=$ $A, B$, there is no free riding in any non-trivial mutually optimal group.

An important implication of Proposition 1 and the corollary is the fact that, if contribution costs are large enough, and as long as connection costs are strictly positive, non-extreme individuals cannot be part of any non-trivial mutually optimal group. The only way moderate

\footnotetext{
${ }^{18}$ Formally, consider any ordered equilibrium in which there are $k^{A} A$-contributions, $k^{B} B$-contributions, and $k^{\varnothing}>0$ individuals who do not contribute. Consider one of the individuals of type $t^{*}$ who contributes. If $U\left(t^{*}, k^{A}, k^{B}-1\right)>U\left(t^{*}, k^{A}-1, k^{B}\right)$, that individual would prefer a group composed of $k^{A}$ agents of type $t^{*}$ and $k^{B}$ agents with taste $t=0$, in which there is a unique equilibrium with $k^{A} A$-contributions and $k^{B} B$ contributions and fewer members overall. Similarly, if $U\left(t^{*}, k^{A}-1, k^{B}\right)>U\left(t^{*}, k^{A}, k^{B}-1\right)$, a group composed of $k^{A}$ agents of taste $t=1$ and $k^{B}$ agents of taste $t^{*}$ would be preferable. If $U\left(t^{*}, k^{A}, k^{B}-1\right)=U\left(t^{*}, k^{A}-1, k^{B}\right)$, the individual would prefer a group of $k^{A}+k^{B}$ agents of type $t^{*}$.
} 
individuals can be part of a non-trivial mutually optimal group (and act as free riders) is when there are no connection costs, at least up to a sufficiently large group size.

\section{Special Cases: Step-function and Linear Connection Costs}

One special case of connection costs corresponds to settings in which, up to a certain capacity, group membership is free. For instance, social friendships may not come at a net cost; however, there is a cap on how many friends one can maintain effectively given time constraints (see, e.g., Dunbar (1992)). Formally, such settings correspond to step-function connection costs: $d(n)=0$ for all $n \leq n^{*}$ and $d(n)=\infty$ for $n>n^{*}{ }^{19}$ In this case $\tilde{n}(c)=n^{*}$ and $c^{*} \equiv \sup \left\{c \mid k_{\max }^{A}(c)+k_{\max }^{B}(c)>n^{*}\right\}$.

In this setting, the restrictions of point (1) of Proposition 1 are automatically satisfied since $d\left(k_{\max }^{A}(c)+k_{\max }^{B}(c)\right)=0$ when $c>c^{*}$ and any group of at most $n^{*}$ members containing $k_{\max }^{A}(c) A$-extremists and $k_{\max }^{B}(c) B$-extremists is mutually optimal. On the other hand, the conditions for mutually optimal groups with homogeneous contributions described in point (2) of Proposition 1 are harsher. Indeed, for $c>c^{*}, d\left(k_{\max }^{x}(c)\right)=0$ for $x=A, B$ and $k^{B}\left(d\left(k_{\max }^{A}(c)\right) ; t\right)=0$ if and only if $t=1$. Similarly, $k^{A}\left(d\left(k_{\max }^{B}(c)\right) ; t\right)=0$ if and only if $t=0$. Therefore, mutually optimal groups with homogenous contributions contain only the polar extremists: either at least $k_{\max }^{A}(c)$ individuals of type $t=1$ or at least $k_{\max }^{B}(c)$ individuals of type $t=0$.

Notice also that when $c$ is very high, any group of $n^{*}$ individuals must contain individuals that ultimately do not contribute, which can be sustained since free-riding members come at no cost (in contrast with the restrictions of Corollary 1 above).

Another special case of connection costs is that of linear connection costs, whereby $D(n)=(n-1) d$ and marginal connection costs are fixed, $d(n)=d$ for all $n \geq 2$. Such

\footnotetext{
${ }^{19}$ Alternatively, we could also assume that $d(n)=M$ for all $n>n^{*}$ for sufficiently large $M$. Furthermore, our analysis would carry through were we to assume $D(n)=0$ for all $n \leq n^{*}$ and $D(n)=M$ for all $n>n^{*}$ for sufficiently large $M$. This is why we slightly abuse labeling and refer to the case analyzed as that of "stepfunction connection costs." In addition, consider the connection cost function $D(1)=0, D(n)=D$ for all $n \leq n^{*}$, and $D(n)=\infty$ for all $n>n^{*}$. That is, being in a group involves a fixed membership fee $D$, and groups have a maximal fixed size $n^{*}$. In this case, for the groups we characterize to remain mutually optimal, one needs to guarantee that the gap between the total utility individuals obtain from being in the group and the utility they obtain by themselves is greater than $D$. As it turns out, any mutually optimal group for $D=0$ is also mutually optimal for sufficiently small $D>0$, unless it involves at least two individuals of which only one contributes.
} 
costs correspond to environments in which connection costs do not depend on the number of individuals. Let $\bar{c}=\sup \left\{c \mid k_{\max }^{A}(c)+k_{\max }^{B}(c) \geq 1\right\}$. Then, $c^{*}=\min \{d, \bar{c}\}$. Suppose that $d$ is not prohibitively high, so that $k_{\max }^{A}(d)+k_{\max }^{B}(d) \geq 1$. In this case, $c^{*}=d$ and as long as the cost of a contribution surpasses the cost of adding a member to the group $(c>d)$, non-trivial mutually optimal groups are composed of extremists. Furthermore, since connection costs are strictly positive for any non-trivial groups, mutually optimal groups will never exhibit free riding in the task-selection stage.

The linear connection cost case allows us to address the analogy between high contribution cost $c$ and low connection cost $d$. If a new technology (for example, E-mail, online platforms for social groups, etc.) makes connection costs decrease relative to contribution costs, agents would like to connect with more people. As described above, the risk of free riding becomes more severe, and reconciled by selecting extremist agents as peers.

Finally, in comparing these two special cases, we observe that step-function connection costs generate a greater collection of mutually optimal groups that exhibit polarization, including ones in which some, potentially moderate, individuals do not ultimately contribute to either task. In contrast, settings with linear connection costs exhibit a richer set of homogenous mutually optimal groups that generate contributions only on one task, containing extremists on either side (whereas step-function connection costs lead to homogenous mutually optimal groups that are comprised only of the polar extremists).

\section{Low Contribution Costs}

As contribution costs decrease, there is less danger of free riding. Accounting for connection costs, it becomes more likely that individuals will want to limit their group size without achieving the maximal attainable volume of contributions. In contrast with the previous section, in this case mutually optimal groups can be composed of contributing moderate members, and the focus shifts to the allocation of these contributions across tasks. In particular, more homogeneous groups, in which members agree on the allocation of contributions to tasks, are likely to emerge as mutually optimal. The following Proposition characterizes mutually optimal groups in the $c \leq c^{*}$ case. 
Proposition 2 (Low $c$ - Mutually Optimal Groups) If $c \leq c^{*}$, non-trivial mutually optimal groups take one of the following forms:

1. (Homogeneous groups) For any $n$ there exists a collection of disjoint intervals $\left\{\mathcal{T}_{l}^{n}\right\}_{l=1}^{h}$ in $[0,1]$ such that $n$ agents of types $t_{1}, \ldots, t_{n}$ form a mutually optimal group if and only if, for some $l=1, . ., h, t_{i} \in \mathcal{T}_{l}^{n}$ for all $i=1, . ., n$.

2. (Mildly polarized groups) A group of $n \leq \tilde{n}(c)$ members such that $n^{A}$ agents have tastes in an interval $I^{A}$, while $n^{B}=n-n^{A}$ agents have tastes in an interval $I^{B}$, where $I^{A} \cap I^{B}=\varnothing$.

Intuitively, in any mutually optimal group members agree on the desirable number of participants. In addition, when contribution costs are sufficiently low relative to the connection costs, if agents are willing to pay the cost of connection, they are certainly willing to pay the cost of contribution in their group. Therefore, agents choose a group that implements their preferred allocation of contributions. It is useful to think of the extreme case in which there are no contribution costs, $c=0$. In that case, any mutually optimal group must be of the sort described in Section 3: for any size $n$ of the group (determined by the connection costs), a group is mutually optimal if and only if all taste parameters in the group belong to the same element of a partition $\left\{T_{k}^{n}\right\}_{k=0}^{n}$ of the interval $[0,1]$. These two restrictions together - all agents agreeing on the size of the group and all agents agreeing on the allocation of contributions within the group - imply the homogeneity of mutually optimal groups as described in part (1) of Proposition 2. Part (2) of the proposition is more of a knife-edge case. It considers situations in which some agents cannot attain their optimal allocation of contributions because of the constraints imposed by the contribution costs. For example, $A$-extremists can implement at most $k_{\max }^{A}(c) A$-contributions. However, at the group selection phase, it is the connection costs $d(n)$ that govern the number of participants in the group and even $A$-extremists (ones for whom $t<1$ ) may be willing to invest in some agents who will contribute to the $B$ task.

We now illustrate the formal construction of mutually optimal groups pertaining to point (1) of Proposition 2. We start by introducing the following Lemma. Recall that for any $t \in[0,1]$, we define $k(s ; t) \equiv k^{A}(s ; t)+k^{B}(s ; t)$ as the total attainable number of contributions 


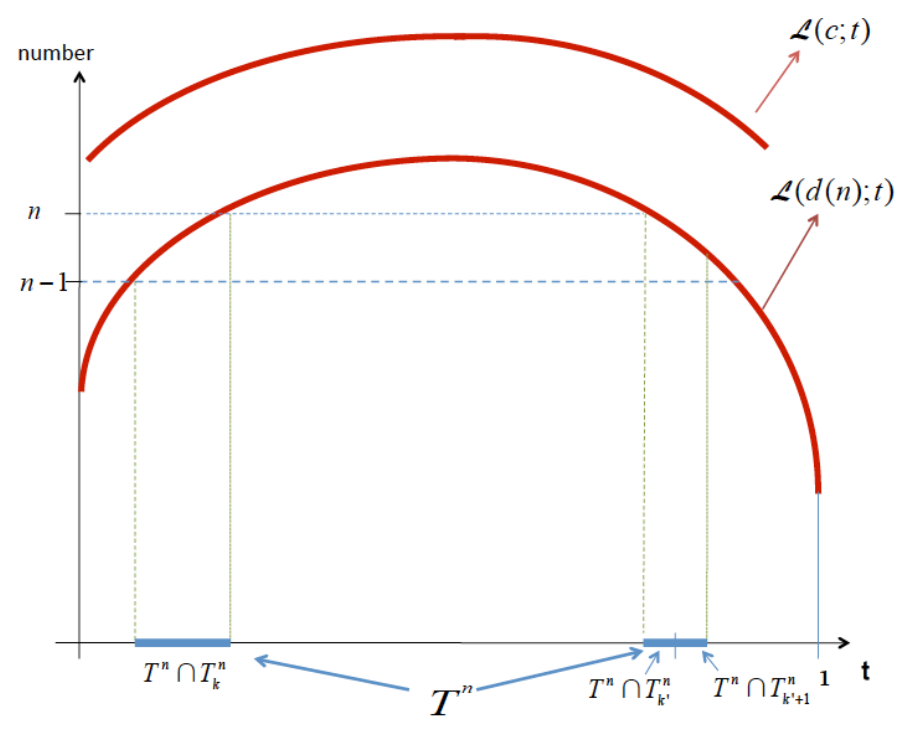

Figure 1: Homogeneous Mutually Optimal Groups (Small c)

at cost $s$ for an agent of taste $t$. Also, recall that $m^{x}(n ; t)$ represents the optimal number of contributions that an individual of taste $t$ would like to allocate to task $x=A, B$ out of a total of $n$ contributions.

Lemma 4 (Low $c$ - Individual Incentives and Group Size) If $n \leq k(c ; t), m^{x}(n ; t) \leq$ $k^{x}(c ; t)$ for $x=A, B$.

Lemma 4 links the size constraint of the group and the personal incentives to make contributions of an individual of taste $t$. As long as group size is sufficiently small (relative to the total attainable number of contributions for an agent of taste $t$ ), the agent engages in contributing when her unconstrained optimal allocation of contributions is implemented. To see why this is the case, suppose that $m^{A}(n ; t)+m^{B}(n ; t)=n \leq k(c ; t)$ but, for instance, $m^{A}(n ; t)>k^{A}(c ; t)$. Then, it must be the case that $m^{B}(n ; t)<k^{B}(c ; t)$. Since $m^{A}(n ; t)$ and $m^{B}(n ; t)$ represent the unconstrained optimal allocation, they are selected in a way that (approximately) equates the marginal returns from contributions toward each task. However, since $m^{B}(n ; t)<k^{B}(c ; t)$, the marginal benefit from the $m^{B}(n ; t)$-th $B$-contribution is greater than $c$. Thus, the marginal benefit from the $m^{A}(n ; t)$-th $A$-contribution should be greater than $c$ as well, in contradiction to $m^{A}(n ; t)>k^{A}(c ; t)$. 
Figure 1 is useful in providing the construction of the groups described in point (1) of Proposition 2. Formally, denote by $K^{A}(s ; t)$ and $K^{B}(s ; t)$ the real numbers achieving equality within the constraints (2) and (3), respectively. For $x=A, B, k^{x}(s ; t)=\left\lfloor K^{x}(s ; t)\right\rfloor$ if $K^{x}(s ; t) \geq 0$ and $k^{x}(s ; t)=0$ if $K^{x}(s ; t)<0$ (by construction, $K^{x}(s ; t)<k_{\max }^{x}(s)+1$ for $x=A, B)$. If $f_{x}(0)-f_{x}(-1)<s, K^{x}(s ; t)=0$. If $f_{x}(h)-f_{x}(h-1)>s$ for all $h$, we denote $K^{x}(s ; t)=k^{x}(s ; t)=\infty$. The number $K^{x}(s ; t)$ captures the point at which an agent of taste $t$ equates the marginal return from an $x$-contribution to the cost $s$. The total attainable number of contributions for an agent of taste $t$, or $k(s ; t)$, is then approximated by the function $\mathcal{L}(s ; t)=\max \left\{K^{A}(s ; t), 0\right\}+\max \left\{K^{B}(s ; t), 0\right\}$. Note that for any given $t, \mathcal{L}(s ; t)$ is decreasing in $s$.

Figure 1 depicts the function $\mathcal{L}(c ; t)$, which, roughly speaking, describes the number of contributions an individual of type $t$ would be willing to acquire at a cost $c$ (were she allowed to make multiple contributions). ${ }^{20}$ Moreover, for $n>\widetilde{n}(c)$. Figure 1 depicts $\mathcal{L}(d(n) ; t)$ which, by definition of $\widetilde{n}(c)$, is below $\mathcal{L}(c ; t)$. In what follows, we consider only the case of groups of size $n>\tilde{n}(c)$, and we describe the construction of homogenous mutually optimal groups of size $n \leq \tilde{n}(c)$ in the Appendix.

Consider first the free contribution case, $c=0$. Since there is no free-rider problem, the marginal values of contributions determine the optimal group size for each individual of type $t$. For any $n>\widetilde{n}(c)$, mutual optimality requires the agents in a group to (approximately) equalize the marginal utilities of the contributions to each other, as well as to the connection cost $d(n)$. Let $\bar{t}$ and $\underline{t}$ be taste parameters that achieve the maximum and minimum of $\mathcal{L}(d(n) ; t)$, respectively. If $n \in\{k(d(n) ; \underline{t}), \ldots, k(d(n) ; \bar{t})\}$, there is a set of taste parameters $T^{n}$ such that all agents with tastes $t \in T^{n}$ desire a total of $n=k(d(n) ; t)$ members in their group. Graphically, looking at Figure $1, T^{n}$ is derived from projecting the integers $n-1$ and $n$ on the $y$-axis onto the $x$-axis via $\mathcal{L}(d(n) ; t)$. By construction, any two agents with tastes $t, t^{\prime} \in T^{n}$ agree that the optimal group size is $n$.

\footnotetext{
${ }^{20}$ In general, the maximum could be achieved by more than one $t$. For the concavity of $\mathcal{L}(c ; t)$ portrayed in Figure 1, it is sufficient to assume that $f_{x}^{\prime \prime \prime} \leq 0$ for $x=A, B$ and $c$ is sufficiently low. As $c$ increases, extreme agents are willing to make a contribution only on the dimension they care most about, and $\mathcal{L}(c ; t)$ becomes piece-wise concave when $f_{x}^{\prime \prime \prime} \leq 0$ for $x=A, B$, as we discuss below.
} 
Now, recall that when groups are of a fixed size $n$ and contributions are free, $c=0$, a group is mutually optimal if and only if all taste parameters in the group belong to the same element of a partition $\left\{T_{k}^{n}\right\}_{k=0}^{n}$ of the interval [0,1]. This is because any two agents with tastes $t, t^{\prime}$ belonging to the same element $T_{k}^{n}$ of the partition agree on the optimal allocation of the $n$ contributions across the two tasks. Therefore, whenever $\mathcal{T}_{k}^{n} \equiv T_{k}^{n} \cap T^{n} \neq \varnothing$, any $n$ agents whose types are selected from $\mathcal{T}_{k}^{n}$ agree on both the group size and the optimal allocation of contributions across the two tasks, and is therefore mutually optimal. It is easy to see that the same construction can be used to characterize mutually optimal groups for positive contribution costs $c$ as long as $n>\widetilde{n}(c)$. Indeed, since $k(c ; t) \geq k(d(n) ; t)$ for all $t$, for any individual in $\mathcal{T}_{k}^{n}$, the condition of Lemma 4 is met, guaranteeing that individual incentives to make contributions are satisfied.

Notice that for high connection costs, $\mathcal{L}(d(2) ; t)<2$ and non-trivial homogenous mutually optimal groups cease to exist. That is, when connection costs are sufficiently high, the intervals described in the first part of Proposition 2 are empty.

Next, we focus our attention on the mildly polarized groups described in part (2) of Proposition 2. For simplicity of exposition, we illustrate here the construction of mildly polarized groups that involve a subgroup of extremists. We describe the construction of any mildly polarized group, which in general involves additional conditions to achieve mutual optimality, in the Appendix. ${ }^{21}$

In particular, we construct mutually optimal groups of size $n \leq \widetilde{n}(c)$ comprised of a subgroup of $A$-extremists that make $k_{\max }^{A}(c) A$-contributions, and a sub-group of $n-k_{\max }^{A}(c)$ more moderate individuals, who all make $B$-contributions. An analogous construction holds for groups with $k_{\max }^{B}(c)$ extremists and $n-k_{\max }^{B}(c)$ more moderate individuals.

Formally, assume that $k_{\max }^{A}(c)$ agents are chosen such that: (i) they are $A$-extremists (i.e., $\left.k^{A}(c ; t)=k_{\max }^{A}(c)\right)$; (ii) their optimal allocation of contributions requires at least $k_{\max }^{A}(c)$

\footnotetext{
${ }^{21}$ The extreme polarization appearing for high $c$ (part (1) of Proposition 1) is not mutually optimal for smaller contribution costs $c$. To see why, consider a polarized group in which $k_{\max }^{A}(c)$ agents are $A$-extremists (and their optimal groups entail $k_{\max }^{A}(c) A$-contributions), and $k_{\max }^{B}(c)$ agents are $B$-extremists (and their optimal groups entail $k_{\max }^{B}(c) B$-contributions). Clearly, when $\widetilde{n}(c)<k_{\max }^{A}(c)+k_{\max }^{B}(c)$, the connection costs are too high for these agents to be in their optimal group. Indeed, the last contributions on both tasks yield, by definition of $k_{\max }^{x}(c)$, a marginal utility of (slightly) less than $c$ to an $x$-extremist. Therefore, say, an $A$-extremist cannot be willing to pay $d\left(k_{\max }^{A}(c)+k_{\max }^{B}(c)\right)>d(\widetilde{n}(c))>c$ to acquire the last $B$-contribution.
} 
contributions to the $A$-task, and (iii) they are willing to pay the connection costs to acquire $k_{\max }^{A}(c) A$-contributions and $n-k_{\max }^{A}(c) B$-contributions. To capture these requirements, we define:

$W^{A}=\left\{t \mid k^{A}(d(n) ; t) \geq k^{A}(c ; t)=k_{\max }^{A}(c), m^{A}(n ; t) \geq k_{\max }^{A}(c)\right.$, and $\left.k^{B}(d(n) ; t)=n-k_{\max }^{A}(c)\right\}$.

Let $k_{\max }^{A}(c)$ agents in the group have taste parameters in the interval $W^{A}$. By construction, these agents are in an optimal group whenever the number of $A$ - and $B$-contributions made is $k_{\max }^{A}(c)$ and $n-k_{\max }^{A}(c)$, respectively.

We choose the remaining $n-k_{\max }^{A}(c)$ agents to satisfy two conditions: (i) they care enough about task $B$ to make $B$-contributions; and (ii) they care enough about task $A$ so that their optimal groups involves $k_{\max }^{A}(c) A$-contributions (they may desire more than $k_{\max }^{A}(c) A$ contributions and be willing to pay the corresponding connection costs for those, but foresee the constraints imposed by contribution costs at the task-selection stage and optimally choose $k_{\max }^{A}(c)$ individuals who are to contribute to the $A$-task). Formally,

$$
Z^{B}=\left\{t \mid k^{B}(d(n) ; t) \geq k^{B}(c ; t)=n-k_{\max }^{A}(c) \text { and } k^{A}(d(n) ; t) \geq k_{\max }^{A}(c)\right\}
$$

If $n<\tilde{n}(c)$, for $k_{\max }^{A}(c)$ agents from $W^{A}$ and $n-k_{\max }^{A}(c)$ agents from $Z^{B}$ to form a mutually optimal group, $Z^{B}$ must contain only $A$-extremists. ${ }^{22}$ For any taste $t$ in $W^{A}$ for which $k^{B}(c ; t)<k^{B}(d(n) ; t), t \notin Z^{B}$. In particular, any $k_{\max }^{A}(c)$ agents from $I^{A} \equiv W^{A} \cap$ $\left\{t \mid k^{B}(c ; t)<n-k_{\max }^{A}(c)\right\}$ and $n-k_{\max }^{A}(c)$ agents from $I^{B} \equiv Z^{B} \cap\left\{t \mid k^{A}(c ; t)=k_{\max }^{A}(c)\right\}$ would constitute a mutually optimal group. By construction, $I^{A}$ and $I^{B}$ are disjoint intervals.

If $n=\tilde{n}(c)$, for $k_{\max }^{A}(c)$ agents from $W^{A}$ and $n-k_{\max }^{A}(c)$ agents from $Z^{B}$ to form a mutually optimal group, agents in $Z^{B}$ need not be $A$-extremists. Following our discussion above, any group of $k_{\max }^{A}(c)$ agents from $I^{A} \equiv W^{A}$ and $n-k_{\max }^{B}(c)$ agents from $I^{B} \equiv Z^{B} \cap$ $\left\{t \mid k^{A}(c ; t)<k_{\max }^{A}(c)\right\}$ is mutually optimal. In addition, any group of $k_{\max }^{A}(c)$ agents from $I^{A} \equiv W^{A} \cap\left\{t \mid k^{B}(c ; t)<n-k_{\max }^{A}(c)\right\}$ and $n-k_{\max }^{B}(c)$ agents from $I^{B} \equiv Z^{B}$ is mutually optimal as well. These satisfy the conditions of the proposition.

\footnotetext{
${ }^{22}$ Otherwise, any moderate agent on both dimensions in $Z^{B}$ would prefer implementing any equilibrium in a group with $k_{\max }^{A}(c)$ agents of taste $t=1$ and $n-k_{\max }^{A}(c)$ other agents of taste $t=0$.
} 
In the Appendix we show that there cannot be mildly polarized groups of size $n>\tilde{n}(c)$, and we also show that the type of groups described in Proposition 2 are the only groups that could be mutually optimal when $c$ is low.

Three remarks are in order. First, with regard to the Corollary of Section 4, it follows from our discussion that a similar result holds for $c \leq c^{*}$. As long as $d\left(k_{\max }^{x}(c)+1\right)>0$ for $x=A, B$, non-trivial mutually optimal groups do not contain free riding agents.

Second, from the construction of the mutually optimal groups described in Proposition 2 , it is apparent that for sufficiently high contribution costs some types may not belong to any non-trivial mutually optimal group. To see this, suppose that for some $t \in[0,1]$ we have $\mathcal{L}(d(n) ; t)=0$ for any $n$. That is, individuals of type $t$ are not willing to incur connection costs in order to associate with others. Clearly, such individuals cannot be part of any non-trivial mutually optimal group. We come back to the description of the set of types that cannot be part of any non-trivial mutually optimal group in the discussion of the linear connection costs case below.

Finally, the results in this section suggest that when innovation improves public good production relative to connection technologies, we should observe more homogeneity in mutually optimal groups. Take residential neighborhoods or cooperatives for example, in which connection is limited by inflexible real estate constraints. In such communities, as public good contributions become cheaper (for example, because of the introduction of carpool lanes that reduce the costs of driving neighbors' kids to different activities or communication technologies that allow efficient event organization, etc.), residents will tend to appear more aligned in their preferences over the allocation of contributions across public goods (for example, soccer tournaments, block parties, religious events, etc.).

\section{Special Cases: Step-function and Linear Connection Costs}

Consider now the special case of step-function connection cost introduced in the previous section. Using the same notation as before, $\tilde{n}(c)=n^{*}$ and the condition $c<c^{*}$ is equivalent to $n^{*}<k_{\max }^{A}(c)+k_{\max }^{B}(c)$. In this setting, mutually optimal groups never entail more than $n^{*}$ individuals, as that would be prohibitively costly through the connection costs. The construction above implies that part (1) of Proposition 2 takes the following form. If there is a 
set $T$ such that $n^{*} \leq k(c ; t)$ for all $t \in T$, then, for any $k=0, \ldots, n^{*}$ for which $T \cap T_{k}^{n^{*}} \neq \varnothing$, a homogeneous group comprised of any $n^{*}$ agents of tastes in $T \cap T_{k}^{n^{*}}$ is mutually optimal.

In this setting, raising the group size $n^{*}$ is analogous to increasing contribution costs in its effect on free-riding incentives. A consequence of our results in the last two sections is then that there is a set of moderate taste parameters such that individuals with those tastes can only be part of mutually optimal groups that are either sufficiently small (in which all members have incentives to contribute) or large (in which the moderate agents free ride on the extremists in the group, who make all contributions).

Consider now settings in which connection costs are linear. Suppose, as before, that $d$, the (fixed) marginal connection cost, is not prohibitively high, so that $k_{\max }^{A}(d)+k_{\max }^{B}(d) \geq 1$. The condition of Proposition 2 is then $c<d$. This setting is useful for illustrating the effects of increasing connection costs. For sufficiently small $d$, the function $\mathcal{L}(d ; t)$ takes the shape depicted in Figure 1, and, by the construction described above, for any $t \in[0,1]$ it is always possible to find a non-trivial mutually optimal group that includes individuals of type $t$ as members. As $d$ increases, extreme agents would like to add contributions only on the task they care most about. For such situations, larger groups correspond to members with either moderate or extreme tastes. Panel (a) of Figure 2 corresponds to a case in which $\mathcal{L}(d ; t)$ becomes piece-wise concave as $d$ increases. ${ }^{23}$ Still, panel (a) of Figure 2 describes a scenario in which individuals of any type $t \in[0,1]$ are included in mutually optimal groups.

As connection costs increase even more, there may be a range of moderate types who do not wish to have any contributions acquired in their group and $\mathcal{L}(d ; t)$ can exhibit a dip for a range of moderate tastes (see panel (b) of Figure 2). In that case, whenever $\mathcal{L}(d ; t)$ is positive for some $t$, either $k^{A}(d ; t)=0$ or $k^{B}(d ; t)=0$. We therefore deduce that when connection costs are very high, the homogenous mutually optimal groups correspond to extremists willing to have only contributions on one dimension made in the group. In addition, panel (b) of Figure 2 illustrates that there is a set of types $t \in[0,1]$ for which $\mathcal{L}(d ; t)=0$. Since these types are not willing to pay any connection costs, the only mutually optimal groups they can belong to are singletons.

\footnotetext{
${ }^{23}$ Formally, it is sufficient to assume that $f_{x}^{\prime \prime \prime}(\cdot) \leq 0$ for $\mathcal{L}(d ; t)$ to be piece-wise concave.
} 


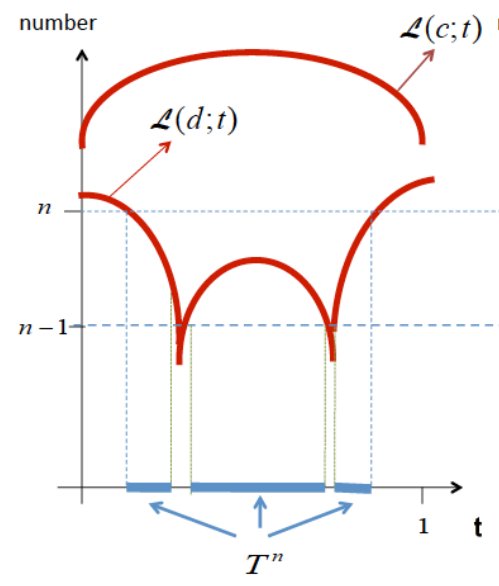

(a)

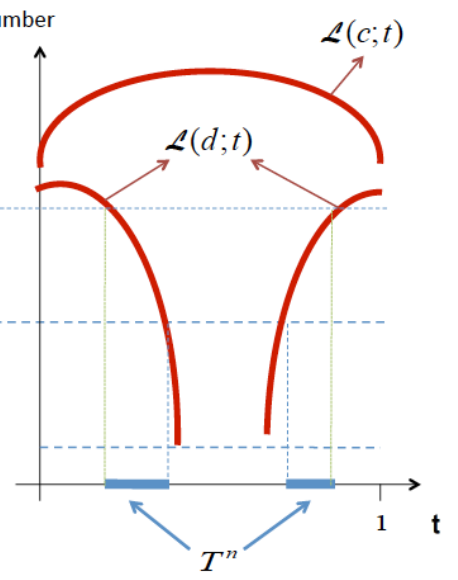

(b)

Figure 2: Homogeneous Mutually Optimal Groups: The Effect of Increasing $d$

To conclude, note that the analysis of the linear connection cost case in the last two sections yields implications on the correlation between group size and composition. In particular, when $d$ is low in relation to $c$, the analysis of the previous section shows that polarized mutually optimal groups emerge, and they include $k_{\max }^{A}(c)+k_{\max }^{B}(c)$ members. On the other hand, as $d$ increases, the analysis in this section applies: mutually optimal groups are mildly polarized or homogeneous and include a number of members always lower than $k_{\max }^{A}(c)+k_{\max }^{B}(c)$. As $d$ increases even more, $\mathcal{L}(d ; t)$ shifts down (see Figure 1 ), and the size of these groups decreases, until they gradually disappear (see Panel (b) in Figure 2). Therefore, our results imply a positive correlation between group size and heterogeneity in the linear case: homogeneous groups tend to be smaller than polarized ones.

\section{Conclusions}

In this paper we address the properties of mutually optimal communities when individuals, who have different tastes, contribute to costly public projects shared with other members of their groups. If contributions to public projects are costly relative to the cost of connecting with other individuals, mutually optimal communities are composed of extreme individuals from both sides of the spectrum. If contributions to public projects are cheap compared to connection costs, mutually optimal groups are formed by individuals who are fairly similar in 
tastes. ${ }^{24}$ Our results suggest that improvements in public project technologies tend to increase homogeneity, while improvements in connection technologies tend to increase heterogeneity in mutually optimal groups.

\footnotetext{
${ }^{24}$ We note that replicating our analysis in a continuous contributions setting would leave the main messages of the paper unchanged, but make the results somewhat starker. Suppose contribution costs are small, and can be distributed continuously across the two tasks. Mutually optimal groups are formed by agents who agree on the group size and the optimal allocation of contributions across the two tasks, and therefore must share exactly the same taste; They are perfectly homogeneous. If contribution costs are large and can be continuously distributed, mutually optimal groups will again be polarized: they will consist of extremists of taste $t=0$ or $t=1$ only.
} 


\section{APPENDIX}

Proof of Lemma 1. Let $t_{1} \geq \ldots \geq t_{n}$. Each agent has to decide whether to acquire an $A$-contribution, a $B$-contribution, or forgo contributing.

To construct an efficient equilibrium in the task-selection game, let $\mu^{A}$ be the maximal integer $h$ such that $t_{h}\left[f_{A}(h)-f_{A}(h-1)\right] \geq c$ (this is inequality (2) in the text). Similarly, let $\mu^{B}$ be the minimal integer $h$ such that $\left(1-t_{h}\right)\left[f_{B}(n-h+1)-f_{B}(n-h)\right] \geq c$ (this is inequality (3) in the text). First, consider the case in which $\mu^{A}+1 \geq \mu^{B}$, so that all agents could be induced to make contributions. We first construct an equilibrium entailing all agents making contributions. We consider an equilibrium as proposed by the Lemma's claim, so that $\tau^{A}=\tau^{B}-1 \equiv \tau^{*}$. Note that if an agent of taste $t$ prefers making an $A$-contribution over a $B$-contribution, so would any agent of taste $t^{\prime}>t$. Similarly, if an agent of taste $t$ prefers making a $B$-contribution, so would any agent of taste $t^{\prime}<t$. In such an equilibrium, the agent with the lowest taste parameter who chooses an $A$-contribution is the agent with taste $t_{\tau^{*}}$. From our tie-breaking rule, it follows that the threshold $\tau^{*}$ is determined as the maximal $\tau \in\{1, \ldots, n\}$ for which agent $\tau$ weakly prefers an $A$-contribution over a $B$-contribution, or for which

$$
U\left(t_{\tau}, \tau, n-\tau\right) \geq U\left(t_{\tau}, \tau-1, n-\tau+1\right)
$$

is satisfied. This inequality is constraint (1) for taste $t_{\tau}$. If (1) is not satisfied for any agent in the group (i.e., $U\left(t_{1}, 0, n\right)>U\left(t_{1}, 1, n-1\right)$ ), then $\tau^{A}=\tau^{*}=0$ and $\tau^{B}=1$ defines an equilibrium. In order to show that choosing $\tau^{*}=0$ if (1) is not satisfied for any positive integer and $\tau^{*}$ as the maximal integer between 1 and $n$ satisfying (1) otherwise defines an equilibrium all that remains to be shown is that incentives to make contributions are satisfied. Notice that for any agent $\tau \leq \tau^{*}$,

$$
\begin{aligned}
U\left(t_{\tau}, \tau, n-\tau\right)-U\left(t_{\tau}, \tau-1, n-\tau+1\right) & =\left[U\left(t_{\tau}, \tau, n-\tau\right)-U\left(t_{\tau}, \tau-1, n-\tau\right)\right] \\
& -\left[U\left(t_{\tau}, \tau-1, n-\tau+1\right)-U\left(t_{\tau}, \tau-1, n-\tau\right)\right] \geq 0
\end{aligned}
$$

and so, the incentives to make a $A$-contribution are greater than those to make a $B$-contribution. Similarly, for agents $\tau>\tau^{*}$, the incentives to make a $B$-contribution are greater than those to make a $A$-contribution. Since $\mu^{A}+1 \geq \mu^{B}$, it follows that the identified profile constitutes an equilibrium. 
Consider now the case in which $\mu^{A}+1<\mu^{B}$, and define $\tau^{A} \equiv \mu^{A}$ and $\tau^{B} \equiv \mu^{B}$. From our definitions of $\mu^{A}$ and $\mu^{B}$, in order to illustrate that the suggested profile constitutes an equilibrium, all that remains to be shown is that an agent making a contribution $x=A, B$ does not prefer to make a contribution $y \neq x$ when all other agents follow the profile. Indeed, suppose that $i \leq \tau^{A}<\tau^{B}$ and observe that

$$
U\left(t_{i}, \tau^{A}, n-\tau^{B}+1\right)-c \geq U\left(t_{i}, \tau^{A}-1, n-\tau^{B}+1\right)>U\left(t_{i}, \tau^{A}-1, n-\tau^{B}+2\right)-c,
$$

where the first inequality follows from inequality (2), and the second from the fact that $\mu^{A}+1<\mu^{B}$. Thus, an agent of taste $t_{i}$ does not profit from deviating to a choice of a $B$-contribution instead of an $A$-contribution. An analogous argument holds for $i \geq \mu^{B}>\mu^{A}$.

Suppose now that there are two equilibria, one of which entails $k^{A} A$-contributions and $k^{B} B$-contributions and one that entails $\tilde{k}^{A} A$-contributions and $\tilde{k}^{B} B$-contributions. We now show that either $k^{A} \leq \tilde{k}^{A}$ and $k^{B} \leq \tilde{k}^{B}$ or $k^{A} \geq \tilde{k}^{A}$ and $k^{B} \geq \tilde{k}^{B}$. Suppose, for instance, that $k^{A}>\tilde{k}^{A}$ and $k^{B}<\tilde{k}^{B}$. This implies that there is an agent with taste $t_{i}$ that in the first equilibrium makes an $A$-contribution, and in the second equilibrium makes no contribution or a $B$-contribution. However, notice that for any such $t_{i}$

$$
\begin{gathered}
U\left(t_{i}, k^{A}, k^{B}\right)-U\left(t_{i}, k^{A}-1, k^{B}\right) \leq U\left(t_{i}, \tilde{k}^{A}+1, \tilde{k}^{B}\right)-U\left(t_{i}, \tilde{k}^{A}, \tilde{k}^{B}\right) \text { and } \\
U\left(t_{i}, k^{A}, k^{B}\right)-U\left(t_{i}, k^{A}-1, k^{B}+1\right) \leq U\left(t_{i}, \tilde{k}^{A}+1, \tilde{k}^{B}-1\right)-U\left(t_{i}, \tilde{k}^{A}, \tilde{k}^{B}\right),
\end{gathered}
$$

in contradiction to $t_{i}$ using a best response in both equilibria. Other cases are shown similarly. Since the equilibrium identified above establishes the maximal volume of contributions, it follows that it is also the most efficient.

Proof of Lemma 2. Let $\widehat{k}_{\max }^{A}$ be the maximal integer $h \geq 2$ such that, no matter how many $w B$-contributions are acquired,

$$
U(1, h, w)-U(1, h-1, w)=\left[f_{A}(h)-f_{A}(h-1)\right] \geq d(h),
$$

and let $\widehat{k}_{\max }^{A}=1$ if there is no such $h$. Define $\widehat{k}_{\max }^{B}$ in the same way. If $\min \left\{k_{\max }^{A}(c), \widehat{k}_{\max }^{A}\right\} \geq 2$, in a group with $\min \left\{k_{\max }^{A}(c), \widehat{k}_{\max }^{A}\right\}$ agents of taste $t=1$, all members are willing to both make 
$A$-contributions and pay the connection costs required to be members. Also, it is immediate to see that these agents are not willing to add additional members to the group. Therefore, such a group is mutually optimal. If $\min \left\{k_{\max }^{A}(c), \widehat{k}_{\max }^{A}\right\}=1$ a mutually optimal group is formed by a single individual with taste $t=1$ who will make one $A$-contribution, and if $k_{\max }^{A}(c)=0$, a mutually optimal group is formed by a single individual with taste $t=1$ who will not contribute. A similar construction applies to mutually optimal groups in which contributions are made only toward task $B$.

Proof of Proposition 1. We first show that the groups identified in the proposition are mutually optimal. Consider first the polarized groups of part (1). Since the group achieves the maximal attainable contributions, no individual desires the addition of an agent. For an $A$-extremist, the benefit of reducing the number of $A$-extremists by one when there are no free-rider is:

$$
d\left(k_{\max }^{A}(c)+k_{\max }^{B}(c)\right) \leq d(\widetilde{n})<c .
$$

Since this $A$-extremist is willing to make an $A$-contribution at a cost of $c$, she is certainly willing to pay $d(\tilde{n})$ to get the additional $A$-contribution. The benefit for an $A$-extremist of type $t$ from reducing the number of $B$-extremists if there are no free riders is $d\left(k_{\max }^{A}(c)+k_{\max }^{B}(c)\right)$. Therefore, as long as she would be willing to make the $k_{\max }^{B}(c)$ 'th $B$-contribution when the cost is $d\left(k_{\max }^{A}(c)+k_{\max }^{B}(c)\right)$, such a reduction would not be beneficial. Therefore, we get the restriction:

$$
k^{B}\left(d\left(k_{\max }^{A}(c)+k_{\max }^{B}(c)\right) ; t\right) \geq k_{\max }^{B}(c) .
$$

In addition, an $A$-extremist who is making an $A$-contribution in the task-selection stage cannot benefit by adding additional $A$-extremists to the group or joining a different group with an identical profile of contributions - in the worst equilibrium, she will still be contributing. Similar arguments follow for any member who is a $B$-extremist.

Following the discussion in the text, when $d(\widetilde{n}+1)=0$, there could be free riders, in addition to the $k_{\max }^{A}(c) A$-extremists and $k_{\max }^{B}(c) B$-extremists.

Consider now the homogenous groups described in part (2) of Proposition 1. As before, none of the $A$-extremists would desire the addition of members of any type. Furthermore, since $d\left(k_{\max }^{A}(c)+k_{\max }^{B}(c)\right) \leq d(\widetilde{n})<c$, eliminating any member of the group would not be beneficial 
either. Analogous arguments pertain to homogeneous groups formed by $B$-extremists.

We now show that there are no non-trivial mutually optimal groups other than those specified in the proposition. The arguments above suggest that an $x$-extremist is in a mutually optimal group only if there are precisely $k_{\max }^{x}(c)-1$ other $x$-extremists in the group as well, $x=A, B$. Therefore, any potentially mutually optimal group other than those specified would include an agent who is not an extremist while $k_{\max }^{A}(c)+k_{\max }^{B}(c) \geq 1$. Suppose there exists such a non-trivial mutually optimal group and consider any ordered equilibrium in that group. If no one contributes, any group member would benefit by switching to a group with one extremist who is willing to contribute. Consider now an ordered equilibrium in which at least one non-extremist member contributes, say an agent of taste $t$, who contributes to task $A$. Let $\tilde{k}^{A}<k_{\max }^{A}(c)$ be the number of $A$-contributions and $\tilde{k}^{B} \leq k_{\max }^{B}(c)$ be the number of $B$ contributions. We first show that for the group to be mutually optimal, it must be the case that $\tilde{k}^{A}=k^{A}(t ; c)$ and $\tilde{k}^{B} \geq k^{B}(t ; c)$. Suppose otherwise. If $\tilde{k}^{A}>k^{A}(t ; c)$, the agent cannot be contributing. If $\tilde{k}^{A}<k^{A}(t ; c)$, the agent would benefit by shifting to a group in which there are $k^{A}(t ; c)-1$ agents with taste $t=1$ and $\tilde{k}^{B}$ agents with taste $t=0$ since

$$
d\left(\tilde{k}^{A}+\tilde{k}^{B}\right) \leq d\left(k_{\max }^{A}(c)+k_{\max }^{B}(c)\right) \leq d(\widetilde{n}(c))<c .
$$

If $\tilde{k}^{B}<k^{B}(t ; c)$, the agent would similarly benefit by shifting to a group with $k^{A}(t ; c)-1$ agents of taste $t=1$ and $k^{B}(t ; c)$ agents of taste $t=0$. Assume then that $\tilde{k}^{A}=k^{A}(t ; c)$ and $\tilde{k}^{B} \geq k^{B}(t ; c)$ and consider a shift to a group in which there are $k^{A}(t ; c)$ agents of taste $t=1$ and $\tilde{k}^{B}$ agents of taste $t=0$. This would, again, come at an additional connection cost that is bounded by $d\left(k_{\max }^{A}(c)+k_{\max }^{B}(c)\right) \leq d(\widetilde{n}(c))<c .^{25}$ In this group, all equilibria would entail our agent not contributing and attaining the same profile of contributions.

Proposition 1A If $c^{*} \neq c^{* *}$ and $c \in\left(c^{*}, c^{* *}\right]$, mutually optimal groups take one of the following forms:

\footnotetext{
${ }^{25}$ The assumption that $d(n) \neq c$ for all $n$ plays a role here. Indeed, suppose $k_{\max }^{A}(c)+k_{\max }^{B}(c)=\tilde{n}-1$ and $d(\tilde{n})=c$. The agent would then be indifferent between adding an $A$-extremist and contributing herself and we could get mutually optimal groups composed of $k^{A}(c ; t)+k^{B}(c ; t)$ individuals of type $t$.
} 
1. A polarized group with $k_{\max }^{A}(c)$ A-extremists, each with a taste $t$ satisfying

$$
k^{B}\left(d\left(k_{\max }^{A}(c)+k_{\max }^{B}(c)\right) ; t\right) \geq k_{\max }^{B}(c)
$$

and $k_{\max }^{B}(c) B$-extremists, each with a taste $t$ satisfying

$$
k^{A}\left(d\left(k_{\max }^{A}(c)+k_{\max }^{B}(c)\right) ; t\right) \geq k_{\max }^{A}(c)
$$

2. A group with $k_{\max }^{x}(c) x$-extremists, each with a taste of $t$ satisfying

$$
k^{y}\left(d\left(k_{\max }^{x}(c)+1\right) ; t\right)=0, x \neq y
$$

3. If $k_{\max }^{A}(c)=1, d\left(1+k_{\max }^{B}(c)\right)=0$, and $d\left(2+k_{\max }^{B}(c)\right)=M$ for sufficiently large $M$, then $k_{\max }^{B}(c)$ agents of taste 0 , and one moderate agent of taste $t \in(0,1)$, such that $k^{A}(c ; t)=0, k^{B}(c ; t)<k_{\max }^{B}(c)$, and

$$
U\left(t, 0, k_{\max }^{B}(c)\right) \geq U\left(t, 1, k_{\max }^{B}(c)-1\right)
$$

Similarly if $k_{\max }^{B}(c)=1$.

Proof of Proposition 1A. The analysis in Proposition 1 carries through as long as $k_{\max }^{A}(c)>$ $1, k_{\max }^{B}(c)>1$, and the classes of groups in points (1) and (2) then comprise all of the mutually optimal groups. Regarding the groups described in point (3), consider $t$ for which $k^{A}(c ; t)=0$. The group consisting of an agent of type $t$ and $k_{\max }^{B}(c)$ agents of taste 0 is mutually optimal, since the agent with non-extreme taste parameter $t$ does not have enough incentives to contribute to one task, even when she is the first to do that. Furthermore, the restriction on $t$ assures that the agent would not benefit from swapping one of the $B$-extremists for an $A$-extremist. Since, $k^{B}(c ; t)<k_{\max }^{B}(c)$, the unique equilibrium in such a group would generate $U\left(t, 1, k_{\max }^{B}(c)-1\right)$. Moreover, the remaining agents have an extreme taste parameter, so have no incentive to make contributions other than to the task they care most about. In addition, since $d(n)=0$ for all $n \leq 1+k_{\max }^{B}(c)$, they pay no additional connection costs for the presence 
of the moderate agent. However, for large enough $M$, no agent in the group desires additional members of any type. The arguments used for the proof of Proposition 1 suggest that the set of mutually optimal groups described in Proposition $1 \mathrm{~A}$ is exhaustive.

Proof of Lemma 4. Suppose that $m^{A}(n ; t)>k^{A}(c ; t)$. Then, it must be the case that $m^{B}(n ; t)<k^{B}(c ; t)$ (otherwise, $n=m^{A}(n ; t)+m^{B}(n ; t)>k^{A}(c ; t)+k^{B}(c ; t)=k(c ; t)$, contrary to our assumption). That is, $k^{B}(c ; t) \geq m^{B}(n ; t)+1$. In particular,

$$
(1-t)\left[f_{B}\left(m^{B}(n ; t)+1\right)-f_{B}\left(m^{B}(n ; t)\right)\right] \geq c
$$

Simple manipulations of the definitions of $m^{A}(n ; t)$ and $m^{B}(n ; t)$ imply

$$
\begin{aligned}
& t\left[f_{A}\left(m^{A}(n ; t)\right)-f_{A}\left(m^{A}(n ; t)-1\right)\right] \\
\geq & (1-t)\left[f_{B}\left(m^{B}(n ; t)+1\right)-f_{B}\left(m^{B}(n ; t)\right)\right] \geq c
\end{aligned}
$$

and $k^{A}(c ; t) \geq m^{A}(n ; t)$, which contradicts our hypothesis. Identical arguments follow if $m^{B}(n ; t)>k^{B}(c ; t)$.

Proof of Proposition 2. We first describe the construction of homogeneous groups described in part (1) of the proposition when $n=\widetilde{n}(c)$ and $n<\widetilde{n}(c)$. For $n=\widetilde{n}(c)$ a construction similar to that discussed in the text for the case of $n<\widetilde{n}(c)$ holds. However, since $d(\widetilde{n}(c))<c$, we need to make sure that any agent within the set $T_{k}^{n} \cap T^{n} \neq \varnothing$ constructed as above is willing to make a contribution. That is, we consider intervals defined as:

$$
\mathcal{T}_{k}^{n} \equiv\left\{t \in T_{k}^{n} \cap T^{n} \neq \varnothing \mid k(c ; t) \geq \widetilde{n}(c)\right\}
$$

If $n<\widetilde{n}(c)$ any non-trivial mutually optimal group must contain extremists. Suppose otherwise, so that fewer than $k_{\max }^{A}(c) A$-contributions and fewer than $k_{\max }^{B}(c) B$-contributions are made. If no one makes a contribution in every ordered equilibrium, any member would benefit from exchanging one of the other members with some extremist. For any ordered equilibrium in which there are $\tilde{k}^{A} A$-contributions and $\tilde{k}^{B} B$-contributions, pick an agent of type $t$ who makes, say, an $A$-contribution. That agent would benefit from switching to a group 
with $n-\tilde{k}^{B}+1$ agents of type $t=1$ and $\tilde{k}^{B}$ agents of type $t=0$. Indeed, if $k^{A}(c ; t)>\tilde{k}^{A}$ the new group is guaranteed to generate an additional $A$-contribution at connection cost lower than $c$ in any equilibrium. If $k^{A}(c ; t)=\tilde{k}^{A}$, then there would be a unique equilibrium in the new group entailing the same profile of contributions, in which our agent is not contributing. Since $d(n+1)<c$ this would be beneficial. Suppose then that the agent with taste $t$ is an extremist, say an $A$-extremist. Since $d(n+1)<c$, it must be the case that in some ordered equilibrium, the volume of $A$-contributions is $k^{A}(c ; t)=k_{\max }^{A}(c)$. Since $\widetilde{n}(c)<k_{\max }^{A}(c)+k_{\max }^{B}(c)$, it follows that in the equilibrium under consideration, the volume $\tilde{k}^{B}$ of $B$-contributions is strictly lower than $k_{\max }^{B}(c)$. Therefore, either all $n$ agents are of type $t=1$ and $d(n)=0$ or all members have taste parameters in the set

$$
\mathcal{T}^{A}=\left\{t \mid k^{A}(c ; t)=k_{\max }^{A}(c), k^{B}(c ; t)=k^{B}(d(n) ; t)=n-k_{\max }^{A}(c)\right\}
$$

which is an interval in $[0,1]$. Notice that from Lemma 4 , any $t \in \mathcal{T}^{A}$ satisfies $k^{x}(c ; t)=m^{x}(n ; t)$ for $x=A, B$. In a group of $n$ members with tastes in $\mathcal{T}^{A}$, for any agent, the addition of either $A$ - or $B$-extremists would lead her to make an $A$-contribution in her worst equilibrium. While any agent could switch to a group in which more $B$-contributions are made, such a switch would be prohibitively costly. Groups as described are therefore mutually optimal. An analogous construction holds for small groups containing $B$-extremists.

We now turn to the mildly polarized groups described in part (2) of the proposition. We first show that there are no mildly polarized groups as described in the text containing $n>\tilde{n}(c)$ members. Indeed, consider groups as such with $k_{\max }^{A}(c) A$-extremist members. When $n>\tilde{n}(c)$, we have $k^{x}(d(n) ; t) \leq k^{x}(c ; t)$ for $x=A, B$. Therefore, for any $t \in W^{A}$, $k^{A}(d(n) ; t)=k^{A}(c ; t)=k_{\max }^{A}(c)$ and $k^{B}(c ; t) \geq k^{B}(d(n) ; t)=n-k_{\max }^{A}(c)$. For any $t \in Z^{B}$, $k^{A}(d(n) ; t)=k^{A}(c ; t)=k_{\max }^{A}(c)$ and $k^{B}(d(n) ; t)=k^{B}(c ; t)=n-k_{\max }^{A}(c)$. In particular, $Z^{B} \subset W^{A}$. Furthermore, any $t \in W^{A} \backslash Z^{B}$ and $t^{\prime} \in Z^{B}$ satisfy $t<t^{\prime}$. Thus, in a group of $k_{\max }^{A}(c)$ agents from $W^{A} \backslash Z^{B}$ and $n-k_{\max }^{A}(c)$ agents from $Z^{B}$, any ordinal equilibrium would entail at least some of the agents from $Z^{B}$ making $A$-contributions, and some agents from $W^{A} \backslash Z^{B}$ making $B$-contributions in contradiction to our construction. It follows that there are no mildly polarized groups including $k_{\max }^{A}(c) A$-extremists for $n>\tilde{n}(c)$. A similar 
argument applies to mildly polarized groups including $k_{\max }^{B}(c) B$-extremists.

We now describe the general construction of mildly polarized groups. In contrast with the homogeneous groups described in part (1) of Proposition 2, in these groups at least some of the agents are not implementing their optimal allocation of contributions across the two tasks. Let $n$ be the total size of the mutually optimal group and select $n^{A}<n$ agents from the set of types $W^{A}$ such that $t \in W^{A}$ if and only if the following conditions are satisfied:

$$
\begin{aligned}
& \text { (i) } \quad m^{A}(n ; t)>n^{A} \\
& \text { (ii) } \quad k^{A}(c ; t)=n^{A} \\
& \text { (iii) } \quad k^{B}(c ; t)<n-n^{A}-1 \\
& \text { (iv) } \quad k^{A}(d(n) ; t) \geq n^{A} \\
& \text { (v) } \quad k^{B}(d(n) ; t) \geq n-n^{A} \\
& \text { (vi) } U\left(t, n^{A}, n-n^{A}\right)-c>U\left(t, n^{A}, n-n^{A}\right)-d(n+1) \text { and } n^{A}<k_{\max }^{A}(c) \\
& \text { or } n^{A}=k_{\max }^{A}(c) \\
& \text { (vii) }(1-t)\left[f_{B}\left(n-n^{A}+1\right)-f_{B}\left(n-n^{A}\right)\right]<d(n+1) \\
& \text { (viii) } U\left(t, n^{A}, n-n^{A}\right)-c \geq \\
& \begin{array}{l}
\max \\
\tilde{k}^{x} \text { x-contributions, } x=A, B, \text { with agent } \\
\text { of taste } t \text { choosing } v \in\{A, B, \varnothing\} \text { is a worst } \\
\text { equilibrium for the agent in a group with } \\
l \leq k_{\max }^{A}(c) A \text {-extremists, } n-1-l \leq k_{\max }^{B}(c) B \text {-extremists }
\end{array}
\end{aligned}
$$

where $(i)$ guarantees that agents in $W^{A}$ are not implementing their optimal allocation, (ii) guarantees that if they add another $A$-contribution they themselves would not have incentives to contribute, $($ iii) guarantees that they are not willing to make a $B$-contribution were one member of the other subgroup to make an $A$-contribution rather than a $B$-contribution (in particular, they are not $B$-extremists), (iv) guarantees that they are willing to connect with all other $A$-contributing members, $(v)$ guarantees that they are willing to connect with the $B$-contributing members, $(v i)$ guarantees that they do not benefit from switching to a group with $n^{A}+1 A$-extremists ${ }^{26}$, (vii) guarantees that they are not willing to add a $B$-extremist to the group, and (viii) assures that they prefer being in this group over any other group of the same size with feasible contributions $\left(\mathbf{1}_{x}\right.$ stands for the indicator function with respect to

\footnotetext{
${ }^{26}$ As discussed in the text, the worst equilibrium in such a group would be beneficial for an agent only if she herself is not an $A$-extremist.
} 
$x)$ and the worst equilibrium being selected. ${ }^{27}$

Note that conditions $(i i)-(v)$ imply together that $c>d(n)$, or $n \leq \widetilde{n}(c)$. Moreover, $(v i)$ simplifies to $c<d(n+1)$, or $n+1>\widetilde{n}(c)$ whenever there are moderate agents in $W^{A}$, in which case $n=\widetilde{n}(c)$. Since the analysis in the text covered the case of mildly polarized groups with extremists, we consider here the case of $W^{A}$ comprised of moderates. By construction, all types $t$ satisfying all the constraints above must belong to an interval - that is, $W^{A}=\left[\underline{t}^{A}, \bar{t}^{A}\right]$. The remaining $\widetilde{n}(c)-n^{A}$ agents are the ones who make $B$-contributions. Notice that a mutually optimal group containing both $A$-extremists and $B$-extremists cannot be smaller than $\widetilde{n}(c)$. It follows from the discussion above and in the text that $n=\tilde{n}(c)$. These agents making $B$-contributions could be selected in two ways: they could be implementing a suboptimal allocation of contributions across the two dimensions analogous to the $A$-contributors (in that case, their types must belong to the corresponding set $W^{B}$ as above with the labels of $A$ and $B$ substituted in the constraints), or they could be implementing their optimal allocation. In the latter case, they are selected from the set $Z^{B}$ of types $t$ such that the following conditions are satisfied:

$$
\begin{array}{ll}
\text { (i) } & m^{A}(\widetilde{n}(c) ; t)=n^{A} \\
\text { (ii) } & k^{B}(c ; t) \geq \widetilde{n}(c)-n^{A} \\
\text { (iii) } & k^{A}(d(\widetilde{n}(c)) ; t) \geq n^{A} \\
\text { (iv) } & k^{B}(d(\widetilde{n}(c)) ; t) \geq \widetilde{n}(c)-n^{A} \\
\text { (v) } & t\left[f_{A}\left(n^{A}+1\right)-f_{A}\left(n^{A}\right)\right]<d(\widetilde{n}(c)+1) \\
\text { (vi) } & (1-t)\left[f_{B}\left(\widetilde{n}(c)-n^{A}+1\right)-f_{B}\left(\widetilde{n}(c)-n^{A}\right)\right]<d(\widetilde{n}(c)+1),
\end{array}
$$

where $(i)$ guarantees that these agents are implementing their optimal allocation, $(i i)$ guarantees that they are all willing to make $B$-contributions, $(i i i)$ guarantees that they are willing to connect with all members of the other subgroup, $(i v)$ guarantees that they are willing to connect with all members of their own subgroup, $(v)$ guarantees that they are not willing to add an $A$-extremist to the group, and $(v i)$ guarantees that they are not willing to add a

\footnotetext{
${ }^{27}$ Suppose the agent contemplates a switch to a group with $\tilde{k}^{x} x$-extremists, $x=A, B$, where $\tilde{k}^{A}+\tilde{k}^{B}=n-1$. If $k^{x}(c ; t) \geq k^{x}+1$ for some $x=A, B, \tilde{k}^{x}+1 x$-contributions and $\tilde{k}^{y} y$-contributions would constitue an equilibrium for $x=A, B, y \neq x$. If $k^{x}(c ; t) \leq k^{x}$ for both $x=A, B$, there would be $\tilde{k}^{x} x$-contributions and $\tilde{k}^{y}$ $y$-contributions with the agent contributing only if $k(c ; t)=k^{x}=k_{\max }^{x}$ for some $x$.
} 
$B$-extremist to the group. Note that $(i v)$ is automatically guaranteed since $c>d(\widetilde{n}(c))$, and that by construction all types $t$ satisfying all the constraints above must belong to an interval - that is, $Z^{B}=\left[\underline{t}^{B}, \bar{t}^{B}\right]$.

An analogous construction holds for a switch in labels of $A$ and $B$ above.

We now show that the classes of groups described in the proposition are the only ones that are mutually optimal when $c<c^{*}$. First, notice that in any mutually optimal group of size $n$ all individuals must agree on the optimal size of the group.

For any mutually optimal group of size $n$ and any member of type $t$ such that $k^{x}(c ; t) \geq$ $m^{x}(n ; t)$ for $x=A, B$, the optimal allocation of contributions for that member is possible and must be accomplished. Our discussion of the proof of the first part of the proposition in the main body of the text illustrates that the corresponding homogeneous groups we describe are the only potentially mutually optimal ones.

Suppose that a group of size $n$ is mutually optimal and contains a member of type $t$ such that, without loss of generality, $k^{A}(c ; t)<m^{A}(n ; t)$. For $c<c^{*}$, it must be the case that $k_{\max }^{A}(c)+k_{\max }^{B}(c) \geq n$. Furthermore, the agent herself must be contributing to some task $x$ in some ordered equilibrium, unless the group consists of agents who are all of taste $t=0$ or all of taste $t=1$ and there are no connection costs required to maintain the group.

Assume first that $k^{B}(c ; t)<m^{B}(n ; t)$. Since $m^{A}(n ; t)+m^{B}(n ; t)=n \leq k_{\max }^{A}(c)+k_{\max }^{B}(c)$, there must be an $x=A, B$ such that $k_{\max }^{x}(c) \geq m^{x}(n ; t)$. Then, it must be the case that the individual makes the $k^{x}(c ; t)^{\prime}$ th $x$-contribution and there are $n-k^{x}(c ; t) y$-contributions made and the construction above implies that the mutually optimal group is mildly polarized.

Assume now that $k^{B}(c ; t) \geq m^{B}(n ; t)$. There are then two cases to consider. Consider first the case $k_{\max }^{A}(c) \geq m^{A}(n ; t)$. In that case, the agent can generate her optimal allocation by joining a group with $m^{A}(n ; t) A$-extremists and $m^{B}(n ; t)$ individuals of type $t$ (that include herself). If $k_{\max }^{A}(c)<m^{A}(n ; t)$, the agent would be optimizing by choosing $k_{\max }^{A}(c)$ $A$-extremists and $n-k_{\max }^{A}(c)$ agents to contribute to the $B$-task, which corresponds to mildly polarized groups described in the text.

From our construction it follows that the set of groups described in Proposition 2 is exhaustive. 


\section{REFERENCES}

[1] Baccara, M., and L. Yariv (2013), "Homophily in Peer Groups," American Economic Journal: Microeconomics, 5(3), 69-96.

[2] Banzhaf, S. and R. Walsh (2008), "Do People Vote with Their Feet? An Empirical Test of the Tiebout's Mechanism," American Economic Review, 98(3), 843-863.

[3] Che, Y-K. and N. Kartik (2009), "Opinions as Incentives," Journal of Political Economy, $\mathbf{1 1 7}(5), 815-860$.

[4] Currarini, S., M. O. Jackson, and P. Pin (2009), "An Economic Model of Friendship: Homophily, Minorities and Segregation," Econometrica, 77(4), 1003-1045.

[5] Dunbar, R. I. M. (1992), "Neocortex size as a constraint on group size in primates," Journal of Human Evolution, 22(6), 469-493.

[6] Ellickson, B., B. Grodal, S. Scotchmer, and W. R. Zame (1999), "Clubs and the Market," Econometrica, 67, 1185-1217.

[7] Gerardi, D. and L. Yariv (2008), "Costly Expertise," American Economic Review, Papers and Proceedings, 98(2), 187-193.

[8] Kets, W. and A. Sandroni (2015a), "A Belief-based Theory of Homophily," mimeo.

[9] Kets, W. and A. Sandroni (2015b), "Challenging Conformity: A Case for Diversity," mimeo.

[10] Ledyard, J. (1995), "Public Goods: A Survey of Experimental Research," in Handbook of Experimental Economics, edited by J. H. Kagel and A. E. Roth, 111-194.

[11] Peski, M. (2008), "Complementarities, Group Formation and Preferences for Similarity," mimeo.

[12] Robbett, A. (2013), "Community Dynamics in the Lab: Congestion, Public Good Provision, and Local Instability," mimeo. 
[13] Robbett, A. (2014), "Local Institutions and the Dynamics of Community Sorting," American Economic Journal: Microeconomics, 6(3), 136-156.

[14] Rhode, P. W. and K. S. Strumpf (2003), "Assessing the Importance of Tiebout Sorting: Local Heterogeneity from 1850 to 1990," The American Economic Review, 93(5), 16481677.

[15] Rosenblat, T. S. and M. M. Mobius (2004), "Getting Closer or Drifting Apart," Quarterly Journal of Economics, 119(3), 971-1009.

[16] Sethi R., and R. Somanathan (2004), "Inequality and Segregation," Journal of Political Economy, 112(6), 1296-1321

[17] Sproull, L. and S. Kiesler (1991), Connections - New Ways of Working in the Networked Organization, MIT Press, Cambridge, MA.

[18] Tiebout, Charles M. (1956), "A Theory of Local Expenditures," The Journal of Political Economy, 64(5), 416-424. 